\title{
EXACT SOLUTIONS OF ONE-DIMENSIONAL TOTAL GENERALIZED VARIATION*
}

\author{
CHRISTIANE PÖSCHL ${ }^{\dagger}$ AND OTMAR SCHERZER ${ }^{\ddagger}$
}

\begin{abstract}
Total generalized variation regularization has been introduced by Bredies, Kunisch, and Pock [K. Bredies, K. Kunisch, and T. Pock, SIAM J. Imaging Sci., 3(3), 492-526, 2010]. This regularization method requires careful tuning of two regularization parameters. The focus of this paper is to derive analytical results, which allow for characterizing parameter settings, which make this method in fact different from total variation regularization (that is the Rudin-Osher-Fatmi model [L.I. Rudin, S. Osher, and E. Fatemi, Phys. D, 60(1-4), 259-268, 1992]) and the second order variation model [O. Scherzer, Computing, 60(1), 1-27, 1998] regularization, respectively. In this paper we also provide explicit solutions of total generalized variation denoising for particular one-dimensional function data.
\end{abstract}

Key words. Fenchel duality, total variation, total generalized variation, bounded Hessian, $G$-norm, convex optimization.

AMS subject classifications. Primary 46N10, Secondary 49M29.

\section{Introduction}

Total generalized variation (TGV) denoising has been introduced in [5]: Given $k \in \mathbb{N}_{0}$ and a function $u^{\delta}: \Omega \rightarrow \mathbb{R}$, where $\Omega \subset \mathbb{R}^{d}$, the method consists in determining

$$
u_{\vec{\lambda}}:=\operatorname{argmin}\left\{\mathcal{G}_{\vec{\lambda}}^{k}(u): u \in L^{2}(\Omega)\right\}
$$

where

$$
\mathcal{G}_{\vec{\lambda}}^{k}(u):=\frac{1}{2} \int_{\Omega}\left(u-u^{\delta}\right)^{2} d x+T G V_{\vec{\lambda}}^{k}(u)
$$

with

$$
\begin{aligned}
& \operatorname{TGV}_{\vec{\lambda}}^{k}(u) \\
= & \sup \left\{\int_{\Omega} u(\nabla \cdot)^{k} \phi d x: \phi \in \mathcal{C}_{c}^{\infty}\left(\Omega, \operatorname{Sym}^{k}\left(\mathbb{R}^{d}\right)\right),\left\|(\nabla \cdot)^{k-l} \phi\right\|_{L^{\infty}} \leq \lambda_{l}, l=1, \ldots, k\right\},
\end{aligned}
$$

and $\operatorname{Sym}^{k}\left(\mathbb{R}^{d}\right)$ denotes the space of symmetric tensors of order $k$ with arguments in $\mathbb{R}^{d}$. There can be imagined several realizations of $\left\|(\nabla \cdot)^{k-l} v\right\|_{L^{\infty}}$ to be implemented: one of them is $\left\|(\nabla \cdot)^{k-l} \phi\right\|_{L^{\infty}}=\sup \left\{\left|(\nabla \cdot)^{k-l} \phi(x)\right|_{l^{2}}: x \in \Omega\right\}$, where $|\cdot|_{l^{2}}$ denotes the Frobenius-norm of a tensor. Note that the definition here is slightly different from the one in [5], where in the original definition, the enumeration of the indices of $\lambda_{i}$ is reversed.

Throughout this paper, for the simplification of notation and considerations, we restrict our attention to the case $k=2$. Consequently, from now on, we omit the superscript $k$ in the TGV-functional.

${ }^{*}$ Received: June 17, 2013; accepted (in revised form): February 21, 2014. Communicated by Luminita Vese.

${ }^{\dagger}$ Department of Mathematics, Alpen Adria Universiät, Klagenfurt, Austria (Christiane.Poeschl@ aau.at).

${ }^{\ddagger}$ Computational Science Center, University of Vienna, Oskar-Morgenstern Platz 1, A-1090 Wien, Austria and Johann Radon Institute of Computational and Applied Mathematics, Austrian Academy of Sciences, Altenberger Straße 69, A-4040 Linz, Austria (Otmar.Scherzer@univie.ac.at). 


\section{EXACT SOLUTION OF ONE-DIMENSIONAL TOTAL GENERALIZED VARIATION}

The goal of this paper is to increase the knowledge about structural properties of TGV-denoising, and to put this method into perspective with total variation and second order total variation regularization by analytical means. This is done in two different ways:

1. The main result of this paper concerns the characterization of the sets of regularization parameters $\vec{\lambda}=\left(\lambda_{1}, \lambda_{2}\right)$ such that the minimizers of $\mathrm{TGV}_{\lambda_{1}, \lambda_{2}}$ either equal total variation minimizers or minimizers of the second order total variation minimization, and to determine sets of parameters where TGV minimization is in fact different from either one of them.

2. We study analytical solutions of simple one-dimensional test-cases, where $d=1, \Omega=(-1,1)$, and $k=2$. In this simple situation TGV-denoising (1.1) simplifies to minimizing the functional

$$
\begin{aligned}
\mathcal{G}: L^{2}(-1,1) & \rightarrow \mathbb{R} \cup\{+\infty\} \\
u & \rightarrow \mathcal{G}(u):=\frac{1}{2} \int_{-1}^{1}\left(u-u^{\delta}\right)^{2} d x+\operatorname{TGV}_{\lambda_{1}, \lambda_{2}}(u) .
\end{aligned}
$$

In the specific one-dimensional situation $\mathrm{TGV}_{\lambda_{1}, \lambda_{2}}$ can be written as

$$
\begin{aligned}
& \operatorname{TGV}_{\lambda_{1}, \lambda_{2}}(u) \\
= & \sup \left\{\int_{-1}^{1} u \phi^{\prime \prime} d x: \phi \in \mathcal{C}_{c}^{\infty}(-1,1),\left\|\phi^{\prime}\right\|_{L^{\infty}} \leq \lambda_{1},\|\phi\|_{L^{\infty}} \leq \lambda_{2}\right\} .
\end{aligned}
$$

Similar to our previous work [15] for total variation minimization and minimization with totally bounded second derivative, it is possible to characterize the minimizers of $\mathrm{TGV}_{\lambda_{1}, \lambda_{2}}$ in a simple manner using Fenchel-duality theory. We show that the minimizers are either equal to $u^{\delta}$ or piecewise affine functions that bend or jump, whenever the first or second primitives of the dual functions attain an extremum.

We then study explicit solutions of TGV-denoising for the basic test data cases

$$
\begin{gathered}
x \rightarrow u^{\delta}(x)=|x|-\frac{1}{2}, \\
x \rightarrow u^{\delta}(x)=\mathbf{1}_{[-1 / 2,1 / 2]}(x)-\frac{1}{2},
\end{gathered}
$$

and

$$
x \rightarrow u^{\delta}(x)=x^{2}-\frac{1}{3} .
$$

For the first two exemplary cases the minimizers of the TGV-functional (1.3) are weighted sums of TV-minimizers and $\mathrm{TV}^{2}$-minimizers. The second example has also been studied in [4]. However, there no complete characterization of the parameter sets have been stated where the $\mathrm{TGV}_{\lambda_{1}, \lambda_{2}}$-minimizer equals either $L^{2}-\mathrm{TV}, L^{2}-\mathrm{TV}^{2}$-minimizers, which is a focus topic of this work.

The outline of this paper is as follows: In Section 2 we introduce preliminary notation and the main definitions. We derive characteristic properties of minimizers of the TGV-denoising problem (in $d$-dimensions) via convex duality theory (sections 
$3,4)$. Later we restrict our attention to the case $d=1$ and show that minimizers are either equal to the data or piecewise affine linear (cf. Section 5). Finally we calculate explicit minimizers for the $\mathrm{TGV}_{\lambda_{1}, \lambda_{2}}$-functional in the case where the data are the absolute value (Section 6), the indicator function (Section 7), or a quadratic polynomial (Section 8), respectively.

\section{Notation}

Let $\Omega \subseteq \mathbb{R}^{d}$ be a bounded, connected domain with Lipschitz boundary. Moreover, let $u^{\delta}: \Omega \rightarrow \mathbb{R}$ belong to $L^{2}(\Omega)$.

For $i \in \mathbb{N}$ we define the following functional:

$$
\begin{aligned}
\mathcal{F}^{i}: L^{2}(\Omega) & \rightarrow \mathbb{R} \cup\{+\infty\}, \\
u & \rightarrow \mathcal{F}^{i}(u):=\frac{1}{2} \int_{\Omega}\left(u-u^{\delta}\right)^{2} d x+\operatorname{TV}_{\lambda_{i}}^{i}(u),
\end{aligned}
$$

where

$$
\operatorname{TV}_{\lambda_{i}}^{i}(u):=\sup \left\{\int_{\Omega} u(\nabla \cdot)^{i} \phi d x: \phi \in \mathcal{C}_{c}^{\infty}\left(\Omega, \operatorname{Sym}^{k}\left(\mathbb{R}^{d}\right)\right),\left\|(\nabla \cdot)^{i} \phi\right\|_{L^{\infty}} \leq \lambda_{i}\right\},
$$

where

$$
\left\|(\nabla \cdot)^{i} v\right\|_{L^{\infty}}=\sup \left\{\left|(\nabla \cdot)^{i} v(x)\right|_{l^{2}}: x \in \Omega\right\},
$$

and $\left|(\nabla \cdot)^{i} \phi\right|_{l^{2}}$ denotes the Frobenius-norm of $(\nabla \cdot)^{i} \phi$.

The minimizer of (2.1) is denoted by $v_{\lambda_{i}}^{i}$. The minimizer of (1.2) is denoted by $u_{\lambda_{1}, \lambda_{2}}$.

Because

$$
\mathrm{TV}_{\lambda_{i}}^{i}(u)=\lambda_{i} \mathrm{TV}^{i}(u)
$$

we see that minimization of the functional $\mathcal{F}^{i}$ from (2.1) is standard $L^{2}-\mathrm{TV}$ minimization with regularization parameter $\lambda_{i}$. $L^{2}-\mathrm{TV}$-minimization has been studied widely in the literature. In the one-dimensional $d=1$ setting it is used for regression (see e.g. [11, 7]); analytical solutions have been calculated for instance in [6]. In image processing, for $d \geq 2, L^{2}-\mathrm{TV}$-regularization it is called the Rudin-OsherFatemi model [16]. Regularization with derivatives of higher order bounded variation has been studied for instance in $[17,19,14,15,18]$. The function spaces of functions of bounded Hessian, and more general convex functionals of functions of bounded Hessian have been introduced and considered in $[8,9]$.

\section{Fenchel duality and applications}

In the following let $\mathcal{H}$ be a Hilbert-space. In this case it is common to identify $\mathcal{H}$ with its dual space and to identify the dual pairing $\left\langle u^{*}, u\right\rangle$ on $\mathcal{H}^{*}$ and $\mathcal{H}$ with the inner product on $\mathcal{H}$. For instance when $\mathcal{H}=L^{2}(\Omega),\left\langle u^{*}, u\right\rangle=\int_{\Omega} u^{*} u d x$.

We start by defining the $*$-number, which is a generalization of the dual norm of a Banach-space, to convex, positively homogeneous functionals.

Definition 3.1. A proper, convex functional $\mathcal{T}: \mathcal{H} \rightarrow \mathbb{R} \cup\{+\infty\}$ is positively homogeneous if there exists some $l=1,2 \ldots$ such that $\mathcal{T}: \mathcal{H} \rightarrow \mathbb{R} \cup\{+\infty\}$ is l-homogeneous, which means that

$$
\mathcal{T}(\lambda u)=|\lambda|^{l} \mathcal{T}(u), \quad \forall \lambda \in \mathbb{R} .
$$


Definition 3.2 (The $*$-number). Let $\mathcal{R}: \mathcal{H} \rightarrow \mathbb{R} \cup\{+\infty\}$ be a positively homogeneous and convex functional. For $u^{*} \in \mathcal{H}$ define

$$
\left\|u^{*}\right\|_{*, \mathcal{R}}:=\sup \left\{\left\langle u^{*}, u\right\rangle: u \in \mathcal{H}, \mathcal{R}(u) \leq 1\right\} .
$$

Moreover define

$$
\mathcal{B}_{\mathcal{R}}^{*}:=\left\{u^{*} \in \mathcal{H}:\left\|u^{*}\right\|_{*, \mathcal{R}} \leq 1\right\}
$$

as the dualball with respect to the $*$-number.

ExAmple 3.1. From (2.3) it follows that

$$
\left\|u^{*}\right\|_{*, \mathrm{TV}_{\lambda_{i}}^{i}}=\frac{1}{\lambda_{i}}\left\|u^{*}\right\|_{*, \mathrm{TV}_{1}^{i}}
$$

Note that according to our definition

$$
\mathrm{TV}_{1}^{i}=\mathrm{TV}^{i}, \quad \forall i \in \mathbb{N}
$$

Lemma 3.3. (see [18, Lemma 4.6]). Let $\mathcal{R}$ be positively homogeneous and set

$$
\mathcal{P}:=\{p \in \mathcal{H}: \mathcal{R}(p)=0\} .
$$

From the assumptions that $\mathcal{R}$ is positively homogeneous and convex, it follows that $\mathcal{P}$ is a linear subspace of $\mathcal{H}$. Denote

$$
\mathcal{P}^{\perp}:=\left\{u^{*} \in \mathcal{H}:\left\langle u^{*}, p\right\rangle=0, p \in \mathcal{P}\right\} .
$$

Then $\left\|u^{*}\right\|_{*, \mathcal{R}}=+\infty$ for all $u^{*} \notin \mathcal{P}^{\perp}$.

Definition 3.4. Assume that $i=1,2, \ldots$ Let $\mathcal{H}=L^{2}(\Omega), \mathcal{R}=T V_{\lambda_{i}}^{i}$, and let $\mathcal{P}^{i}$ be the set of polynomials of order $i-1$. Then

$$
\mathcal{H}^{i}:=\left\{u \in L^{2}(\Omega): \int_{\Omega} u(x) x^{j} d x=0,|j|=0,1, \ldots, i-1, j \in \mathbb{N}_{0}^{d}\right\}=\mathcal{P}^{i \perp} .
$$

Remark 3.2. Because $C_{c}^{\infty}(\Omega)$ is dense in $L^{2}(\Omega)$, and $\Omega$ is assumed to be bounded, it follows that

$$
\mathcal{H}^{i}:=\overline{\left\{u \in C_{c}^{\infty}(\Omega): \int_{\Omega} u(x) x^{j} d x=0,|j|=0,1, \ldots, i-1, j \in \mathbb{N}_{0}^{d}\right\}} .
$$

The following lemma is a direct consequence of Lemma 3.3 and the above definition.

LEMMA 3.5.

- $T V_{\lambda_{i}}^{i}, i=1,2$ : For all $u^{*} \notin \mathcal{H}^{i},\left\|u^{*}\right\|_{*, T V_{\lambda_{i}}^{i}}=+\infty$.

- $T G V$-functional: For all $u^{*} \notin \mathcal{H}^{2},\left\|u^{*}\right\|_{*, T G V_{\lambda_{1}, \lambda_{2}}}=+\infty$. 
The definitions of the $\|\cdot\|_{*, \mathrm{TV}_{\lambda_{i}}^{i}}$-norms are similar to those in Meyer's book [12]; see also [1]). The difference is that there $\Omega=\mathbb{R}^{d}$ is considered, and the elements of the space $L^{2}\left(\mathbb{R}^{d}\right)$ satisfy natural boundary conditions at $\infty$. Because we consider bounded domains $\Omega$ we restrict attention to the subspaces $\mathcal{H}^{i}$ rather than to $L^{2}$, as in Meyer's book. Another possibility, instead of factorizing out polynomials, is to consider boundary conditions on the bounded domain $\Omega$, which has been realized in [2].

The Fenchel dual of a proper functional $\mathcal{S}: \mathcal{H} \rightarrow \mathbb{R} \cup\{+\infty\}$ is defined as

$$
\begin{aligned}
\mathcal{S}^{*}: \mathcal{H} & \rightarrow \mathbb{R} \cup\{+\infty\} . \\
u^{*} & \mapsto \mathcal{S}^{*}:=\sup _{u \in \mathcal{H}}\left\{\left\langle u^{*}, u\right\rangle-\mathcal{S}(u)\right\} .
\end{aligned}
$$

The following results can be found in [10]; see also [18].

\section{REMARK 3.3.}

- Let $\mathcal{T}$ be 1-homogeneous, then the Fenchel dual function is a characteristic function of a convex set $\mathcal{C}^{*}$. That is,

$$
\mathcal{T}^{*}\left(u^{*}\right)=\chi_{\mathcal{C}^{*}}\left(u^{*}\right)= \begin{cases}0, & \text { for } u^{*} \in \mathcal{C}^{*}, \\ +\infty, & \text { else. }\end{cases}
$$

In particular, for $\mathcal{T}$ 1-homogeneous,

$$
\mathcal{C}^{*}=\mathcal{B}_{\mathcal{T}}^{*}
$$

- Let $\mathcal{S}, \mathcal{R}$ be convex and proper functionals defined on $\mathcal{H}$. Denote by $\hat{u}$ a minimizer of the functional $u \rightarrow \mathcal{S}(u)+\mathcal{R}(u)$ and denote by $\hat{u}^{*}$ a minimizer of the functional $u^{*} \rightarrow \mathcal{S}^{*}\left(u^{*}\right)+\mathcal{R}^{*}\left(-u^{*}\right)$. Then the extremality conditions hold:

$$
\hat{u}^{*} \in \partial \mathcal{S}(u) \quad \text { and } \quad-\hat{u}^{*} \in \partial \mathcal{R}(u)
$$

EXAMPLE 3.4.

1. The dual functional of

$$
\begin{aligned}
\mathcal{S}: L^{2}(\Omega) & \rightarrow \mathbb{R} \\
u & \rightarrow \frac{1}{2} \int_{\Omega}\left(u-u^{\delta}\right)^{2} d x
\end{aligned}
$$

is given by

$$
\begin{aligned}
\mathcal{S}^{*}: L^{2}(\Omega) & \rightarrow \mathbb{R}, \\
u^{*} & \rightarrow \frac{1}{2} \int_{\Omega} u^{* 2} d x+\int_{\Omega} u^{*} u^{\delta} d x .
\end{aligned}
$$

In the case of the quadratic functional the extremality condition (3.5) for a minimizer shows

$$
\hat{u}^{*}=\hat{u}-u^{\delta} .
$$


2. Let

$$
\mathcal{R}=\mathrm{TV}_{\lambda_{i}}^{i}: L^{2}(\Omega) \rightarrow \mathbb{R} \cup\{+\infty\},
$$

which is 1 -homogeneous. Then

$$
\mathcal{R}^{*}\left(u^{*}\right)=\sup _{u \in L^{2}(\Omega)}\left\{\int_{\Omega} u^{*} u d x-\mathrm{TV}_{\lambda_{i}}^{i}(u)\right\} \underset{(2.3)}{=} \chi_{\mathcal{B}_{\lambda_{i}}^{*} \mathrm{TV}^{i}}\left(u^{*}\right),
$$

where the characteristic function is 0 on the closed unit ball $\mathcal{C}^{*}=\mathcal{B}_{\lambda_{i} \mathrm{TV}^{i}}^{*}$ and $+\infty$ else.

3. Let

$$
\mathcal{R}=\mathrm{TGV}_{\lambda_{1}, \lambda_{2}}: L^{2}(\Omega) \rightarrow \mathbb{R} \cup\{+\infty\},
$$

which is 1-homogeneous. Thus $\mathcal{R}^{*}\left(u^{*}\right)=\chi_{\mathcal{B}_{\mathrm{TGV}_{\lambda_{1}, \lambda_{2}}^{*}}}$.

\section{Regularization methods with 1-homogeneous regularizers}

In the following we derive some properties of regularization functionals with $\mathcal{S}$ from (3.6) and 1-homogeneous regularizers $\mathcal{R}$. We denote

$$
u \rightarrow \mathcal{E}(u):=\mathcal{S}(u)+\mathcal{R}(u) .
$$

Remark 4.1. From (3.4) we know that

$$
\mathcal{R}^{*}=\chi_{\mathcal{B}_{\mathcal{R}}^{*}}
$$

Then the extremality condition (3.8) guarantees that $\hat{u}^{*}=\hat{u}-u^{\delta} \in \mathcal{B}_{\mathcal{R}}^{*}$ and from Fenchel-duality theory we see that

$$
\begin{aligned}
& \frac{1}{2} \int_{\Omega}\left(\hat{u}-u^{\delta}\right)^{2} d x+\mathcal{R}(\hat{u}) \\
= & \inf \{\mathcal{S}(u)+\mathcal{R}(u)\} \\
= & -\inf \left\{\mathcal{S}^{*}\left(u^{*}\right)+\mathcal{R}^{*}\left(-u^{*}\right)\right\} \\
= & -\mathcal{S}^{*}\left(\hat{u}^{*}\right)-\underbrace{\chi_{\mathcal{B}_{\mathcal{R}}^{*}}\left(-\hat{u}^{*}\right)}_{=0} \\
\underbrace{}_{(3.7)} & -\int_{\Omega} \frac{1}{2}\left(\hat{u}-u^{\delta}\right)^{2}+u^{\delta}\left(\hat{u}-u^{\delta}\right) d x .
\end{aligned}
$$

In summary we have shown that

$$
\begin{aligned}
\left\|\hat{u}^{*}\right\|_{*, \mathcal{R}} & \leq 1, \\
\mathcal{R}(\hat{u}) & =-\int_{\Omega}\left(\hat{u}-u^{\delta}\right)^{2}+u^{\delta}\left(\hat{u}-u^{\delta}\right) d x=-\int_{\Omega}\left(\hat{u}-u^{\delta}\right) \hat{u} d x .
\end{aligned}
$$

- (4.2), applied to $L^{2}-\mathrm{TV}$-minimization, shows that $v_{\lambda_{i}}^{i}$, the minimizer of $\mathcal{F}^{i}$ (see (2.1)), satisfies

$$
\left\|v_{\lambda_{i}}^{i *}\right\|_{*, \mathrm{TV}_{\lambda_{i}}^{i}} \leq 1 \text { and } \mathrm{TV}_{\lambda_{i}}^{i}\left(v_{\lambda_{i}}^{i}\right)=-\int_{\Omega}\left(v_{\lambda_{i}}^{i}-u^{\delta}\right) v_{\lambda_{i}}^{i} d x
$$

$\left\|v_{\lambda_{i}}^{i *}\right\|_{*, \mathrm{TV}_{\lambda_{i}}^{i}} \leq 1$ implies that $v_{\lambda_{i}}^{i *} \in \mathcal{H}^{i}$, because according to Lemma 3.3 $\left\|v_{\lambda_{i}}^{i *}\right\|_{*, \mathrm{TV}_{\lambda_{i}}^{i}}$ would be $+\infty$ otherwise. This, in particular, means that if $u^{\delta} \in \mathcal{H}^{i}$, then also $v_{\lambda_{i}}^{i} \in \mathcal{H}^{i}$. 
- (4.2), applied to $\mathrm{TGV}_{\lambda_{1}, \lambda_{2}}$-minimization, shows that $u_{\lambda_{1}, \lambda_{2}}$, the minimizer of (1.3), satisfies

$$
\left\|u_{\lambda_{1}, \lambda_{2}}^{*}\right\|_{*, \mathrm{TGV}_{\lambda_{1}, \lambda_{2}}} \leq 1 \text { and } \operatorname{TGV}_{\lambda_{1}, \lambda_{2}}\left(u_{\lambda_{1}, \lambda_{2}}\right)=-\int_{\Omega}\left(u_{\lambda_{1}, \lambda_{2}}-u^{\delta}\right) u_{\lambda_{1}, \lambda_{2}} d x .
$$

Now $\left\|u_{\lambda_{1}, \lambda_{2}}^{*}\right\|_{*, \operatorname{TGV}_{\lambda_{1}, \lambda_{2}}} \leq 1$ implies that $u_{\lambda_{1}, \lambda_{2}}^{*} \in \mathcal{H}^{2}$ (Lemma 3.3). Because $u_{\lambda_{1}, \lambda_{2}}^{*}=u_{\lambda_{1}, \lambda_{2}}-u^{\delta}, u^{\delta} \in \mathcal{H}^{2}$ implies that also $u_{\lambda_{1}, \lambda_{2}} \in \mathcal{H}^{2}$, hence by assuming that $u^{\delta} \in \mathcal{H}^{2}$ we can equivalently minimize $\mathcal{G}$ over $\mathcal{H}^{2}$ instead of $L^{2}(\Omega)$.

Lemma 4.1. If $u$ satisfies (4.2) then $u$ minimizes $\mathcal{E}$.

In particular

- $u$ minimizes $\mathcal{F}^{i}$ if and only if $-\int_{\Omega}\left(u-u^{\delta}\right) u d x=T V_{\lambda_{i}}^{i}(u)$ and $u-u^{\delta}$ in $\mathcal{B}_{T V_{\lambda_{i}}^{i}}^{*}$,

- $u$ minimizes the $T G V$-functional if and only if $-\int_{\Omega}\left(u-u^{\delta}\right) u d x=$ $T G V_{\lambda_{1}, \lambda_{2}}(u)$ and $u-u^{\delta}$ in $\mathcal{B}_{T G V_{\lambda_{1}, \lambda_{2}}^{*}}^{*}$.

Proof. We prove the lemma by contradiction. Assume that $u$ satisfies the assumptions of the lemma but is not a minimizer of $\mathcal{E}$. Then there exists some $v \neq u$ such that $v$ minimizes $\mathcal{E}$ and $\mathcal{E}(v)<\mathcal{E}(u)$. From (4.4) it then follows that

$$
\mathcal{R}(v)=-\int_{\Omega}\left(v-u^{\delta}\right) v d x
$$

Therefore, from the assumption that $u$ satisfies (4.2), we see that

$$
\begin{aligned}
& \frac{1}{2} \int_{\Omega}\left(v-u^{\delta}\right)^{2} d x-\int_{\Omega}\left(v-u^{\delta}\right) v d x \\
= & \frac{1}{2} \int_{\Omega}\left(v-u^{\delta}\right)^{2} d x+\mathcal{R}(v) \\
< & \frac{1}{2} \int_{\Omega}\left(u-u^{\delta}\right)^{2} d x+\mathcal{R}(u) \\
= & \frac{1}{2} \int_{\Omega}\left(u-u^{\delta}\right)^{2} d x-\int_{\Omega}\left(u-u^{\delta}\right) u d x,
\end{aligned}
$$

so that

$$
-\frac{1}{2} \int_{\Omega} v^{2} d x<-\frac{1}{2} \int_{\Omega} u^{2} d x
$$

The dual functional of a convex, 1-homogeneous function $\mathcal{R}$ is the characteristic function of $\mathcal{B}_{\mathcal{R}}^{*}$ (cf. Remark 4.1). The Fenchel-duality theorem (see e.g. [10]) states that $v^{*}:=v-u^{\delta}$ minimizes the functional $w^{*} \rightarrow \mathcal{S}^{*}\left(w^{*}\right)$ over $\mathcal{B}_{\mathcal{R}}^{*}$, where $\mathcal{S}^{*}$ is as in (3.7), such that we have now

$$
\begin{aligned}
\mathcal{S}^{*}\left(v-u^{\delta}\right) & =\int_{\Omega}\left(\frac{1}{2}\left(v-u^{\delta}\right)^{2}+\left(v-u^{\delta}\right) u^{\delta}\right) d x \\
& \leq \mathcal{S}^{*}\left(u-u^{\delta}\right)=\int_{\Omega}\left(\frac{1}{2}\left(u-u^{\delta}\right)^{2}+\left(u-u^{\delta}\right) u^{\delta}\right) d x .
\end{aligned}
$$

The inequality above simplifies to

$$
\frac{1}{2} \int_{\Omega} v^{2} d x \leq \frac{1}{2} \int_{\Omega} u^{2} d x
$$


such that we obtain a contradiction to (4.5). Hence the assumption that $v \neq u$ is a minimizer of $\mathcal{E}$ was wrong.

Lemma 4.2. Assume that $\mathcal{R}$ is a 1-homogeneous functional on $\mathcal{H}$. Then $u_{\min } \equiv 0$ minimizes $\mathcal{E}$ if and only if $\left\|u^{\delta}\right\|_{*, \mathcal{R}} \leq 1$.

Proof.

- 0 minimizes $\mathcal{E} \Rightarrow\left\|u^{\delta}\right\|_{*, \mathcal{R}} \leq 1$ : If $u_{\min } \equiv 0$, then $u_{\min }^{*}=-u^{\delta}$ and the extremality conditions from Remark 4.1 state that $u_{\min }^{*} \in \mathcal{B}_{\mathcal{R}}^{*}$. This means that $\left\|u_{\min }^{*}\right\|_{*, \mathcal{R}} \leq 1$ and consequently $\left\|u^{\delta}\right\|_{*, \mathcal{R}} \leq 1$.

- $\left\|u^{\delta}\right\|_{*, \mathcal{R}} \leq 1 \Rightarrow u_{\min } \equiv 0$ : We prove this implication by contradiction. Assume therefore that $\left\|u^{\delta}\right\|_{*, \mathcal{R}} \leq 1$ and that $u_{\min } \not \equiv 0$ minimizes $\mathcal{E}$. This, in particular, means that $\mathcal{R}\left(u_{\text {min }}\right)<+\infty$. Then from (4.2) it follows that

$$
-\int_{\Omega} u_{\min }\left(u_{\min }-u^{\delta}\right) d x=\mathcal{R}\left(u_{\min }\right) \geq\left\|u^{\delta}\right\|_{*, \mathcal{R}} \mathcal{R}\left(u_{\min }\right) .
$$

Rearranging the terms and dividing by $\mathcal{R}\left(u_{\min }\right)$ shows that

$$
\begin{aligned}
-\int_{\Omega} \frac{u_{\min }^{2}}{\mathcal{R}\left(u_{\min }\right)} d x+\int_{\Omega} \frac{u_{\min }}{\mathcal{R}\left(u_{\min }\right)} u^{\delta} d x & \geq\left\|u^{\delta}\right\|_{*, \mathcal{R}} \\
& =\sup \left\{\int_{\Omega} u^{\delta} \phi d x: \mathcal{R}(\phi) \leq 1\right\} .
\end{aligned}
$$

Because, by assumption, $0 \neq u_{\min } \in L^{2}(\Omega)$, we also have

$$
\int_{\Omega} \frac{u_{\min }^{2}}{\mathcal{R}\left(u_{\min }\right)} d x>0
$$

This, together with (4.6), shows that

$$
\begin{aligned}
\sup \left\{\int_{\Omega} u^{\delta} \phi d x: \mathcal{R}(\phi) \leq 1\right\} & \underbrace{\geq}_{\phi=\frac{u_{\min }}{\mathcal{R}\left(u_{\min }\right)}} \int_{\Omega} \frac{u_{\min }}{\mathcal{R}\left(u_{\min }\right)} u^{\delta} d x \\
& >\left\|u^{\delta}\right\|_{*, \mathcal{R}} \\
& =\sup \left\{\int_{\Omega} u^{\delta} \phi d x: \mathcal{R}(\phi) \leq 1\right\}
\end{aligned}
$$

hence we obtain a contradiction to the assumption $u_{\min } \neq 0$.

\section{EXAMPLE 4.2 .}

1. If $\mathcal{R}=\mathrm{TV}_{\lambda_{i}}^{i}$, then from Lemma 4.2 it follows that $v_{\lambda_{i}}^{i} \equiv 0$ if and only if $\left\|u^{\delta}\right\|_{*, \mathrm{TV}_{\lambda_{i}}^{i}} \leq 1$

2. $\mathrm{TGV}_{\lambda_{1}, \lambda_{2}}$-minimization: Choose $\mathcal{R}=\mathrm{TGV}_{\lambda_{1}, \lambda_{2}}$. Then from Lemma 4.2 it follows that $u_{\lambda_{1}, \lambda_{2}} \equiv 0$ if and only if $\left\|u^{\delta}\right\|_{*, \mathrm{TGV}_{\lambda_{1}, \lambda_{2}}} \leq 1$.

These results are similar with those in [12], where $T V$-minimization of functions on $\Omega=\mathbb{R}^{d}$ have been considered. 


\section{Extremal properties and solutions of 1D-TGV}

In the following we consider the case $d=1$ and $\Omega=(-1,1)$. We derive some characteristic properties of the minimizers $u_{\lambda_{1}, \lambda_{2}}$ of the $\operatorname{TGV}_{\lambda_{1}, \lambda_{2}}$-functional $\mathcal{G}_{\lambda_{1}, \lambda_{2}}$, defined in (1.2).

Below, by some basic considerations, it is possible to identify sets of parameters $\vec{\lambda}=\left(\lambda_{1}, \lambda_{2}\right)$ for which $u_{\lambda_{1}, \lambda_{2}}$ equals some $v_{\lambda_{i}}^{i}, i=1,2$.

For $d=1$, the dual-norm $\|\cdot\|_{*, \mathrm{TV}_{\lambda_{i}}^{i}}, i=1,2,\left\|u^{*}\right\|_{*, \mathrm{TGV}_{\lambda_{1}, \lambda_{2}}}$, respectively, can be easily calculated via integration. To see this, let

$$
\begin{aligned}
\sigma^{0}\left[u^{*}\right](x) & :=u^{*}(x), \\
\sigma^{1}\left[u^{*}\right](x) & :=\int_{-1}^{x} u^{*}(t) d t, \\
\sigma^{i+1}\left[u^{*}\right](x) & :=\int_{-1}^{x} \sigma^{i}\left[u^{*}\right](t) d t, \quad \forall u^{*} \in L^{2}(-1,1) .
\end{aligned}
$$

Lemma 5.1. Let $\Omega=(-1,1)$. Then for all $i=1,2, \ldots$,

$$
\begin{aligned}
\Psi^{i} & :=\left\{\psi \in C_{c}^{\infty}(-1,1): \sigma^{j}[\psi](1)=0, j=1,2, \ldots, i-1\right\} \\
& =\mathcal{H}^{i} \cap C_{c}^{\infty}(-1,1) .
\end{aligned}
$$

Moreover,

$$
\overline{\Psi^{i}}=\mathcal{H}^{i}
$$

Proof. If $u^{*} \in \mathcal{H}^{i} \cap C_{c}^{\infty}(-1,1)$, then

$$
\int_{-1}^{1} u^{*} x^{j} d x=0, \quad \forall j=0,1, \ldots i-1
$$

For fixed $i$ we prove by an inductive argument that for $u^{*} \in \mathcal{H}^{i} \cap C_{c}^{\infty}(-1,1)$ also $u^{*} \in$ $\Psi^{i}$.

- If $j=1$, then $u^{*} \in \mathcal{H}^{i}$ implies that

$$
\sigma^{j}\left[u^{*}\right](1)=\sigma^{1}\left[u^{*}\right](1)=\int_{-1}^{1} u^{*} d x=\int_{-1}^{1} u^{*} 1 d x=0 .
$$

- Let $2 \leq j \leq i-1$ and assume that $\sigma^{k}\left[u^{*}\right](1)=0$ for $k=0,1, \ldots, j-1$. Then

$$
\sigma^{j}\left[u^{*}\right](1)=\int_{-1}^{1} \sigma^{j-1}\left[u^{*}\right] d x=-\int_{-1}^{1} \sigma^{j-2}\left[u^{*}\right] x d x+\sigma^{j-1}\left[u^{*}\right](1) .
$$

The right hand side vanishes because $u^{*} \in \mathcal{H}^{i} \cap C_{c}^{\infty}(-1,1)$ and by the induction assumption.

The reverse direction can be performed with an analogous induction argument. (5.3) follows from Remark 3.2 and the fact that $\mathcal{H}^{i}$ is closed in $L^{2}(-1,1)$. 
180 EXACT SOLUTION OF ONE-DIMENSIONAL TOTAL GENERALIZED VARIATION

Using this lemma we are able to derive a characterization of the TV-seminorm via $\sigma^{i}$ : For all $u \in L^{2}(-1,1)$ we have the identity

$$
\begin{aligned}
& \operatorname{TV}_{1}^{i}(u) \\
= & \sup \left\{\int_{-1}^{1} u \phi^{i} d x: \phi \in \mathcal{C}_{c}^{\infty}(-1,1),\|\phi\|_{L^{\infty}} \leq 1\right\} \\
= & \sup \left\{\int_{-1}^{1} u \phi^{i} d x: \phi^{i} \in \mathcal{C}_{c}^{\infty}(-1,1), \phi^{j}( \pm 1)=0, j=0,1, \ldots, i-1,\|\phi\|_{L^{\infty}} \leq 1\right\} \\
= & \sup \left\{\int_{-1}^{1} u \psi d x: \psi \in \Psi^{i} \cap \mathcal{C}_{c}^{\infty}(-1,1),\left\|\sigma^{i}[\psi]\right\|_{L^{\infty}} \leq 1\right\} \\
\underbrace{}_{(5.2)} & \sup \left\{\int_{-1}^{1} u \phi d x: \phi \in \mathcal{H}^{i} \cap C_{c}^{\infty}(-1,1),\left\|\sigma^{i}(\phi)\right\|_{L^{\infty}} \leq 1\right\} .
\end{aligned}
$$

Using (5.3), $\overline{\Psi^{i}}=\mathcal{H}^{i}$, and the fact that $u \rightarrow\left\|\sigma^{i}[u]\right\|_{L^{\infty}}$ is lower semi-continuous with respect to the $L^{2}$-norm, it follows that

$$
\begin{aligned}
\operatorname{TV}_{\lambda_{i}}^{i}(u) & =\lambda_{i} \operatorname{TV}_{1}^{i}(u) \\
& =\sup \left\{\int_{-1}^{1} u \phi d x: \phi \in \mathcal{H}^{i},\left\|\sigma^{i}(\phi)\right\|_{L^{\infty}} \leq \lambda_{i}\right\} .
\end{aligned}
$$

From [15, Theorem 5.1] we then get an equivalent characterization of $\mathrm{TV}_{\lambda_{i}}^{i}$ :

$$
T V_{1}^{i}(u)=\sup \left\{\int_{-1}^{1} u \psi d x: \psi \in \mathcal{H}^{i},\|\psi\|_{*, T V_{1}^{i}} \leq 1\right\} .
$$

In an analogous way, we can rewrite the $\mathrm{TGV}_{\lambda_{1}, \lambda_{2}}$-functional

$$
\begin{aligned}
& \operatorname{TGV}_{\lambda_{1}, \lambda_{2}}(u) \\
= & \sup \left\{\int_{-1}^{1} u \phi^{\prime \prime} d x: \phi \in \mathcal{C}_{c}^{\infty}(-1,1),\left\|\phi^{\prime}\right\|_{L^{\infty}} \leq \lambda_{1},\|\phi\|_{L^{\infty}} \leq \lambda_{2}\right\} \\
= & \sup \left\{\int_{-1}^{1} u \phi d x: \phi \in \mathcal{H}^{2},\left\|\sigma^{1}[\phi]\right\|_{L^{\infty}} \leq \lambda_{1},\left\|\sigma^{2}[\phi]\right\|_{L^{\infty}} \leq \lambda_{2}\right\} .
\end{aligned}
$$

Lemma 5.2. Let $d=1$ and $\Omega=(-1,1)$.

- For $u \in \mathcal{H}^{2}$ and $i=1,2$, we have

$$
T G V_{\lambda_{1}, \lambda_{2}}(u) \leq T V_{\lambda_{i}}^{i}(u)
$$

- For $u^{*} \in \mathcal{H}^{2}$ and $i=1,2$, we have

$$
\left\|u^{*}\right\|_{*, T V_{\lambda_{i}}^{i}} \leq\left\|\sigma^{i}\left[u^{*}\right]\right\|_{L^{\infty}},\left\|u^{*}\right\|_{*, T V_{\lambda_{i}}^{i}} \leq\left\|u^{*}\right\|_{*, T G V_{\lambda_{1}, \lambda_{2}}} .
$$

As a consequence

$$
\mathcal{B}_{T G V_{\lambda_{1}, \lambda_{2}}}^{*} \subset \mathcal{B}_{T V_{\lambda_{1}}^{1}}^{*} \cap \mathcal{B}_{T V_{\lambda_{2}}^{2}}^{*}
$$

On the other hand, if $u^{*}$ satisfies

$$
\left\|\sigma^{i}\left(u^{*}\right)\right\|_{L^{\infty}} \leq \lambda_{i}, i=1,2 \text {, then } u^{*} \in \mathcal{B}_{T G V_{\lambda_{1}, \lambda_{2}}}^{*} .
$$


Moreover,

$$
T G V_{\lambda_{1}, \lambda_{2}}(u)=\sup \left\{\int_{\Omega} u \psi d x: \psi \in \mathcal{H}^{2}:\|\psi\|_{*, T G V_{\lambda_{1}, \lambda_{2}}} \leq 1\right\} .
$$

Proof. First, we note that for every $\rho \in \mathcal{H}^{2}$

$$
\int_{\Omega} \rho p d x=0, \quad \forall p \in \mathcal{P}^{1} .
$$

- From (5.6) it follows that

$$
\begin{aligned}
& \operatorname{TGV}_{\lambda_{1}, \lambda_{2}}(u) \\
= & \sup \left\{\int_{-1}^{1} u \phi d x: \phi \in \mathcal{H}^{2},\left\|\sigma^{1}[\phi]\right\|_{L^{\infty}} \leq \lambda_{1},\left\|\sigma^{2}[\phi]\right\|_{L^{\infty}} \leq \lambda_{2}\right\} \\
\leq & \sup \left\{\int_{-1}^{1} u \phi d x: \phi \in \mathcal{H}^{2},\left\|\sigma^{2}[\phi]\right\|_{L^{\infty}} \leq \lambda_{2}\right\} \\
= & \operatorname{TV}_{\lambda_{2}}^{2}(u), \quad \forall u \in \mathcal{H}^{2} .
\end{aligned}
$$

Moreover, from (5.5) and (5.11) it follows for all $u \in \mathcal{H}^{2}$ that

$$
\begin{aligned}
& \sup \left\{\int_{-1}^{1} u \phi d x: \phi \in \mathcal{H}^{1},\left\|\sigma^{1}[\phi]\right\|_{L^{\infty}} \leq \lambda_{1}\right\} \\
= & \sup \left\{\int_{-1}^{1} u \phi d x: \phi \in \mathcal{H}^{1} \cap \mathcal{P}^{2 \perp},\left\|\sigma^{1}[\phi]\right\|_{L^{\infty}} \leq \lambda_{1}\right\} \\
= & \sup \left\{\int_{-1}^{1} u \phi d x: \phi \in \mathcal{H}^{2},\left\|\sigma^{1}[\phi]\right\|_{L^{\infty}} \leq \lambda_{1}\right\} \\
= & \operatorname{TV}_{\lambda_{1}}^{1}(u), \quad \forall u \in \mathcal{H}^{2} .
\end{aligned}
$$

Thus

$$
\operatorname{TGV}_{\lambda_{1}, \lambda_{2}}(u) \leq \operatorname{TV}_{\lambda_{1}}^{1}(u), \quad \forall u \in \mathcal{H}^{2} .
$$

- Because $\mathrm{TV}_{\lambda_{i}}^{i}$ and $\mathrm{TGV}_{\lambda_{1}, \lambda_{2}}$ are lower semi-continuous on $L^{2}(-1,1)$ it follows that

$$
\begin{aligned}
& \left\{u \in \mathcal{H}^{i}: \operatorname{TV}_{\lambda_{i}}^{i}(u) \leq 1\right\} \\
= & \overline{\left\{u \in \mathcal{C}_{c}^{\infty}(-1,1) \cap \mathcal{H}^{i}: \operatorname{TV}_{\lambda_{i}}^{i}(u) \leq 1\right\}} \\
\subset & \overline{\left\{u \in \mathcal{C}_{c}^{\infty}(-1,1) \cap \mathcal{H}^{i}: \operatorname{TGV}_{\lambda_{1}, \lambda_{2}}(u) \leq 1\right\}} \\
= & \left\{u \in \mathcal{H}^{i}: \operatorname{TGV}_{\lambda_{1}, \lambda_{2}}(u) \leq 1\right\}, \quad \forall i=1,2 .
\end{aligned}
$$

Therefore, from (5.11) it follows that

$$
\begin{aligned}
\left\|u^{*}\right\|_{*, \mathrm{TV}_{\lambda_{i}}^{i}} & =\sup \left\{\int_{\Omega} u^{*} u d x: u \in \mathcal{H}^{i}, \mathrm{TV}_{\lambda_{i}}^{i}(u) \leq 1\right\} \\
& =\sup \left\{\int_{\Omega} u^{*} u d x: u \in \mathcal{H}^{2}, \operatorname{TV}_{\lambda_{i}}^{i}(u) \leq 1\right\}, \quad \forall u^{*} \in \mathcal{H}^{2} .
\end{aligned}
$$


This, together with (5.12), implies that

$$
\begin{aligned}
\left\|u^{*}\right\|_{*, \mathrm{TV}_{\lambda_{i}}^{i}} & =\sup \left\{\int_{\Omega} u^{*} u d x: u \in \mathcal{H}^{2}, \operatorname{TV}_{\lambda_{i}}^{i}(u) \leq 1\right\} \\
& \leq \sup \left\{\int_{\Omega} u^{*} u d x: u \in \mathcal{H}^{2}, \operatorname{TGV}_{\lambda_{1}, \lambda_{2}}(u) \leq 1\right\} \\
& =\left\|u^{*}\right\|_{*, \mathrm{TGV}_{\lambda_{1}, \lambda_{2}}}, \quad \forall u^{*} \in \mathcal{H}^{2} .
\end{aligned}
$$

The definition of the $*$-number shows that

$$
\left\|u^{*}\right\|_{*, \mathrm{TV}_{1}^{i}}=\sup \left\{\int_{-1}^{1} u^{*} u d x: u \in \mathcal{H}^{i}: \operatorname{TV}_{1}^{i}(u)=1\right\} .
$$

For all $u \in \mathcal{H}^{i}$ satisfying $\operatorname{TV}_{1}^{i}(u)=1$ we have

$$
\begin{aligned}
1=\operatorname{TV}_{1}^{i}(u) & =\sup \left\{\int_{-1}^{1} u \phi^{*} d x: \phi^{*} \in \mathcal{H}^{i},\left\|\sigma^{i}\left(\phi^{*}\right)\right\|_{L^{\infty}} \leq 1\right\} \\
& =\sup \left\{\int_{-1}^{1} u \frac{\phi^{*}}{\left\|\sigma^{i}\left(\phi^{*}\right)\right\|_{L^{\infty}}} d x: \phi^{*} \in \mathcal{H}^{i}\right\} .
\end{aligned}
$$

Choosing $\phi^{*}=u^{*}$ then gives

$$
\int_{-1}^{1} u u^{*} d x \leq\left\|\sigma^{i}\left(u^{*}\right)\right\|_{L^{\infty}}, \quad \forall u \in \mathcal{H}^{i} \text { with } \operatorname{TV}_{1}^{i}(u)=1
$$

This shows that

$$
\left\|u^{*}\right\|_{*, \mathrm{TV}_{1}^{i}} \leq\left\|\sigma^{i}\left(u^{*}\right)\right\|_{L^{\infty}}, \quad \forall u^{*} \in \mathcal{H}^{i}
$$

- To prove (5.9) we use the definition of the $*$-norm:

$$
\begin{aligned}
& \left\|u^{*}\right\|_{*, \operatorname{TGV}_{\lambda_{1}, \lambda_{2}}} \\
= & \sup \left\{\int_{-1}^{1} u^{*} u d x: u \in \mathcal{H}^{2}, \operatorname{TGV}_{\lambda_{1}, \lambda_{2}}(u) \leq 1\right\}, \\
& \operatorname{TGV}_{\lambda_{1}, \lambda_{2}}(u) \\
= & \sup \left\{\int_{\Omega} u \phi^{\prime \prime} d x: \phi \in \mathcal{C}_{c}^{\infty}(-1,1),\left\|\phi^{\prime}\right\|_{L^{\infty}} \leq \lambda_{1},\left\|\phi^{\prime \prime}\right\|_{L^{\infty}} \leq \lambda_{2}\right\} .
\end{aligned}
$$

The function $\phi=\sigma^{2}\left[u^{*}\right]$ satisfies

i $u^{*}=\phi^{\prime \prime}$,

ii by assumption,

$$
\left\|\phi^{\prime}\right\|_{L^{\infty}}=\left\|\sigma^{1}\left(u^{*}\right)\right\|_{L^{\infty}} \leq \lambda_{1},\|\phi\|_{L^{\infty}}=\left\|\sigma^{2}\left(u^{*}\right)\right\|_{L^{\infty}} \leq \lambda_{2}
$$

If $\mathrm{TGV}_{\lambda_{1}, \lambda_{2}}(u) \leq 1$, it then follows that

$$
\int_{-1}^{1} u u^{*} d x \leq \operatorname{TGV}_{\lambda_{1}, \lambda_{2}}(u) \leq 1
$$

Taking the supremum with respect to $u$ then shows that $\left\|u^{*}\right\|_{*, \operatorname{TGV}_{\lambda_{1}, \lambda_{2}}} \leq 1$. 
On the other hand, according to (5.13), $u^{*} \in \mathcal{B}_{\mathrm{TGV}_{\lambda_{1}, \lambda_{2}}}^{*}$ implies that

$$
\max \left\{\left\|u^{*}\right\|_{*, \mathrm{TV}_{\lambda_{1}}^{1}},\left\|u^{*}\right\|_{*, \mathrm{TV}_{\lambda_{2}}^{2}}\right\} \leq 1 .
$$

Because

$$
\left\|u^{*}\right\|_{*, \mathrm{TV}_{\lambda_{i}}^{i}}=\frac{1}{\lambda_{i}}\left\|\sigma^{i}\left[u^{*}\right]\right\|_{L^{\infty}}, \quad \forall u^{*} \in \mathcal{B}_{\mathrm{TGV}_{\lambda_{1}, \lambda_{2}}}^{*},
$$

it then follows that

$$
\left\|\sigma^{i}\left[u^{*}\right]\right\|_{L^{\infty}} \leq \lambda_{i}, \quad \forall u^{*} \in \mathcal{B}_{\mathrm{TGV}_{\lambda_{1}, \lambda_{2}}}^{*}, \forall i=1,2 .
$$

- As a consequence

$$
\begin{aligned}
\operatorname{TGV}_{\lambda_{1}, \lambda_{2}}(u) & =\sup \left\{\int_{\Omega} u \phi^{\prime \prime} d x: \phi \in \mathcal{C}_{c}^{\infty}(\Omega),\left\|\phi^{\prime}\right\|_{L^{\infty}} \leq \lambda_{1},\left\|\phi^{\prime \prime}\right\|_{L^{\infty}} \leq \lambda_{2}\right\} \\
& =\sup \left\{\int_{\Omega} u \psi d x: \psi \in \mathcal{H}^{2},\|\psi\|_{*, \operatorname{TGV}_{\lambda_{1}, \lambda_{2}}} \leq 1\right\} .
\end{aligned}
$$

Lemma 5.3. For

$$
\lambda_{2} \geq\left\|\sigma^{2}\left[v_{\lambda_{1}}^{1 *}\right]\right\|_{L^{\infty}}
$$

we have $v_{\lambda_{1}}^{1}=u_{\lambda_{1}, \lambda_{2}}$ and $T G V_{\lambda_{1}, \lambda_{2}}\left(v_{\lambda_{1}}^{1}\right)=T V_{\lambda_{1}}^{1}\left(v_{\lambda_{1}}^{1}\right)$. On the other hand, if

$$
\lambda_{1} \geq\left\|\sigma^{1}\left[v_{\lambda_{2}}^{2 *}\right]\right\|_{L^{\infty}},
$$

then $v_{\lambda_{2}}^{2}=u_{\lambda_{1}, \lambda_{2}}$ and $T G V_{\lambda_{1}, \lambda_{2}}\left(v_{\lambda_{2}}^{2}\right)=T V_{\lambda_{2}}^{2}\left(v_{\lambda_{2}}^{2}\right)$.

Proof. We only prove the first assertion. The proof of the second assertion is analogous, and therefore omitted.

We summarize two properties of $v_{\lambda_{1}}^{1}$ :

- By assumption $\left\|\sigma^{2}\left[v_{\lambda_{1}}^{1 *}\right]\right\|_{L^{\infty}} \leq \lambda_{2}$.

- Because $v_{\lambda_{1}}^{1}$ minimizes (2.1) it follows from [15] that $\sigma_{1}\left[v_{\lambda_{1}}^{1 *}\right] \leq \lambda_{1}$.

From (5.9) it then follows that

$$
v_{\lambda_{1}}^{1 *} \in \mathcal{B}_{\mathrm{TGV}_{\lambda_{1}, \lambda_{2}}^{*}}^{*} .
$$

Because $v_{\lambda_{1}}^{1}$ is the minimizer of the TV-functional $\mathcal{F}^{1}$ it follows from Lemma 4.1 and (5.4) that

$$
\begin{aligned}
-\int_{-1}^{1}\left(v_{\lambda_{1}}^{1}-u^{\delta}\right) v_{\lambda_{1}}^{1} d x & =\operatorname{TV}_{\lambda_{1}}^{1}\left(v_{\lambda_{1}}^{1}\right) \\
& =\sup \left\{\int_{-1}^{1} v_{\lambda_{1}}^{1} v^{*} d x: v^{*} \in \mathcal{H}^{1} \cap \mathcal{C}_{c}^{\infty}(-1,1),\left\|\sigma^{1}\left(v^{*}\right)\right\|_{L^{\infty}} \leq \lambda_{1}\right\} .
\end{aligned}
$$

Moreover, because $v_{\lambda_{1}}^{1} \in \mathcal{B}_{\mathrm{TGV}_{\lambda_{1}, \lambda_{2}}}^{*}$, we have

$$
0=\mathrm{TGV}_{\lambda_{1}, \lambda_{2}}^{*}\left(v_{\lambda_{1}}^{1 *}\right)=\sup _{u \in L^{2}(-1,1)}\left\{\int_{-1}^{1} v_{\lambda_{1}}^{1 *} u d x-\mathrm{TGV}_{\lambda_{1}, \lambda_{2}}(u)\right\},
$$




\section{EXACT SOLUTION OF ONE-DIMENSIONAL TOTAL GENERALIZED VARIATION}

and in particular for the test function $u=-v_{\lambda_{1}}^{1}$,

$$
-\int_{-1}^{1}\left(v_{\lambda_{1}}^{1}-u^{\delta}\right) v_{\lambda_{1}}^{1} d x=-\int_{-1}^{1} v_{\lambda_{1}}^{1 *} v_{\lambda_{1}}^{1} d x \leq \operatorname{TGV}_{\lambda_{1}, \lambda_{2}}\left(-v_{\lambda_{1}}^{1}\right)=\operatorname{TGV}_{\lambda_{1}, \lambda_{2}}\left(v_{\lambda_{1}}^{1}\right)
$$

This, together with Lemma 5.2 and (5.17), shows that

$$
-\int_{-1}^{1}\left(v_{\lambda_{1}}^{1}-u\right) v_{\lambda_{1}}^{1} d x \leq \operatorname{TGV}_{\lambda_{1}, \lambda_{2}}\left(v_{\lambda_{1}}^{1}\right) \leq \operatorname{TV}_{\lambda_{1}}^{1}\left(v_{\lambda_{1}}^{1}\right)=-\int\left(v_{\lambda_{1}}^{1}-u\right) v_{\lambda_{1}}^{1} d x
$$

and therefore in particular

$$
-\int_{-1}^{1}\left(v_{\lambda_{1}}^{1}-u\right) v_{\lambda_{1}}^{1} d x=\operatorname{TGV}_{\lambda_{1}, \lambda_{2}}\left(v_{\lambda_{1}}^{1}\right)
$$

Applying Lemma 4.1 with (5.16) and (5.19) shows that $v_{\lambda_{1}}^{1}$ also minimizes the $\mathrm{TGV}_{\lambda_{1}, \lambda_{2}}$-regularization (1.3).

Definition 5.4. We define

$$
\Lambda:=\left\{\left(\lambda_{1}, \lambda_{2}\right): 1<\left\|\sigma^{1}\left(v_{\lambda_{2}}^{2 *}\right)\right\|_{L^{\infty}} \text { and } 1<\left\|\sigma^{2}\left(v_{\lambda_{1}}^{1 *}\right)\right\|_{L^{\infty}}\right\} .
$$

Corollary 5.5. If $\left(\lambda_{1}, \lambda_{2}\right) \in\left[\left\|\sigma^{1}\left(u^{\delta}\right)\right\|_{L^{\infty}}+\infty\right) \times\left[\left\|\sigma^{2}\left(u^{\delta}\right)\right\|_{L^{\infty}},+\infty\right)$, then $u_{\lambda_{1}, \lambda_{2}} \equiv 0$.

Proof. Because of (3.1) and (5.7) we have

$$
\lambda_{1} \geq\left\|\sigma^{1}\left(u^{\delta}\right)\right\|_{L^{\infty}} \geq\left\|u^{\delta}\right\|_{*, \mathrm{TV}_{1}^{1}} \text { and } \lambda_{2} \geq\left\|\sigma^{2}\left(u^{\delta}\right)\right\|_{L^{\infty}} \geq\left\|u^{\delta}\right\|_{*, \mathrm{TV}_{1}^{2}} .
$$

Therefore, from Example 4.2 and Lemma 4.2 it follows that $v_{\lambda_{2}}^{2}=v_{\lambda_{1}}^{1} \equiv 0$.

Using Lemma 5.3, $v_{\lambda_{2}}^{2}=v_{\lambda_{1}}^{1}=u_{\lambda_{1}, \lambda_{2}}$, and therefore the assertion follows.

LEMma 5.6. Let $u_{\lambda_{1}, \lambda_{2}}^{*}$ be the minimizer of $u^{*} \rightarrow \mathcal{S}^{*}\left(u^{*}\right)+T G V_{\lambda_{1}, \lambda_{2}}^{*}\left(-u^{*}\right)$.

1. Then, on each connected component of

$$
B:=\left\{x:\left|\sigma^{1}\left[u_{\lambda_{1}, \lambda_{2}}^{*}\right](x)\right|<\lambda_{1} \text { and }\left|\sigma^{2}\left[u_{\lambda_{1}, \lambda_{2}}^{*}\right](x)\right|<\lambda_{2}\right\},
$$

$\left.u_{\lambda_{1}, \lambda_{2}}(x)\right|_{B}$ is a polynomial of maximal degree 1 .

2. If there exists an interval $A$ such that either $\left|\sigma^{1}\left[u_{\lambda_{1}, \lambda_{2}}^{*}\right](x)\right|=\lambda_{1}$ for all $x \in A$ or $\left|\sigma^{2}\left[u_{\lambda_{1}, \lambda_{2}}^{*}\right](x)\right|=\lambda_{2}$ for all $x \in A$, then $u_{\lambda_{1}, \lambda_{2}}=u^{\delta}$ on $A$.

3. Jump Condition: If there exists $x_{0} \in(-1,1)$ and $\epsilon>0$ such that

$$
\begin{aligned}
\sigma^{1}\left[u_{\lambda_{1}, \lambda_{2}}^{*}\right]\left(x_{0}\right) & =\lambda_{1} \text { and } \\
\left|\sigma^{1}\left[u_{\lambda_{1}, \lambda_{2}}^{*}\right](x)\right| & <\lambda_{1}, \quad \forall x \in\left(x_{0}-\epsilon, x_{0}+\epsilon\right) \backslash\left\{x_{0}\right\},
\end{aligned}
$$

then there exist constants $c_{1}$, and $d_{1} \leq d_{2}$, such that

$$
u_{\lambda_{1}, \lambda_{2}}(x)= \begin{cases}c_{1} x+d_{1}, & x \in\left(x_{0}-\epsilon, x_{0}\right), \\ c_{1} x+d_{2}, & x \in\left(x_{0}, x_{0}+\epsilon\right) .\end{cases}
$$

If, instead of the first condition in (5.20), $\sigma^{1}\left[u_{\lambda_{1}, \lambda_{2}}^{*}\right]\left(x_{0}\right)=-\lambda_{1}$ holds, then $u_{\lambda_{1}, \lambda_{2}}$ satisfies (5.21) but $d_{2} \leq d_{1}$. 
4. Bending Condition: If there exists $x_{0} \in(-1,1)$ and some $\epsilon>0$ such that

$$
\begin{aligned}
\sigma^{2}\left[u_{\lambda_{1}, \lambda_{2}}^{*}\right]\left(x_{0}\right) & =\lambda_{2}, \\
\left|\sigma^{2}\left[u_{\lambda_{1}, \lambda_{2}}^{*}\right](x)\right| & <\lambda_{2}, \quad \forall x \in\left(x_{0}-\epsilon, x_{0}+\epsilon\right) \backslash\left\{x_{0}\right\},
\end{aligned}
$$

then

$$
u_{\lambda_{1}, \lambda_{2}}(x)= \begin{cases}c_{1} x+d_{1}, & x \in\left(x_{0}-\epsilon, x_{0}\right), \\ c_{2} x+d_{2}, & x \in\left(x_{0}, x_{0}+\epsilon\right)\end{cases}
$$

is continuous at $x_{0}$, and $c_{2} \leq c_{1}$, where the later condition we refer to as negative bending.

If instead of the first condition in (5.22), $\sigma^{2}\left[u_{\lambda_{1}, \lambda_{2}}^{*}\right]\left(x_{0}\right)=-\lambda_{2}$ holds, then the function is positively bending, i.e., $c_{1} \leq c_{2}$.

(1)
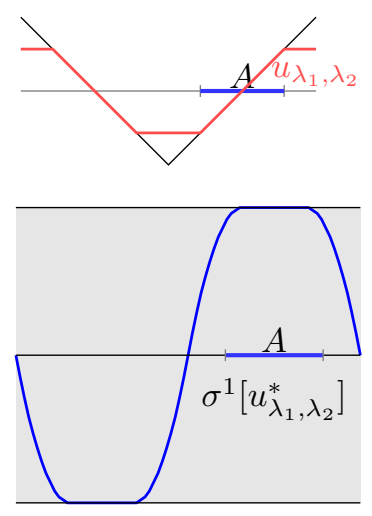
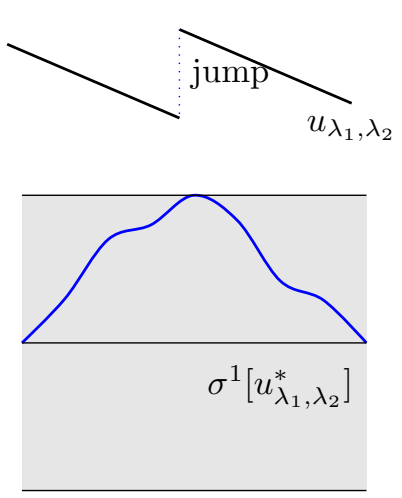

(4)
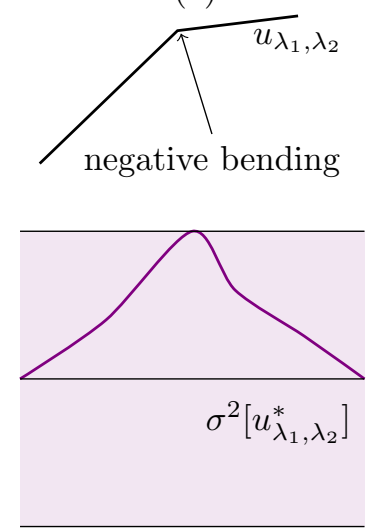

FIG. 5.1. Illustration of Lemma 5.7 and 5.6.

Proof. Recall that if $w^{*} \notin \mathcal{H}^{2}$, then $w^{*} \notin \mathcal{B}_{\mathrm{TGV}_{\lambda_{1}, \lambda_{2}}}$, hence in the following we restrict our attention to $w^{*} \in \mathcal{H}^{2}$. The Kuhn-Tucker condition $-u_{\lambda_{1}, \lambda_{2}} \in \partial \mathcal{R}^{*}\left(u_{\lambda_{1}, \lambda_{2}}^{*}\right)$ guarantees that

$$
\mathcal{R}^{*}\left(v^{*}\right)-\mathcal{R}^{*}\left(u_{\lambda_{1}, \lambda_{2}}^{*}\right)+\int_{-1}^{1} u_{\lambda_{1}, \lambda_{2}}\left(v^{*}-u_{\lambda_{1}, \lambda_{2}}^{*}\right) d x \geq 0, \quad \forall v^{*} \in \mathcal{H}^{2} .
$$

In particular, for $w^{*} \in \mathcal{B}_{\mathrm{TGV}_{\lambda_{1}, \lambda_{2}}}^{*}$ we have

$$
\int_{-1}^{1} u_{\lambda_{1}, \lambda_{2}}\left(w^{*}-u_{\lambda_{1}, \lambda_{2}}^{*}\right) d x \geq 0
$$

Item 1: Let $(a, b)$ be an open interval such that

$$
\left|\sigma^{i}\left[u_{\lambda_{1}, \lambda_{2}}^{*}\right](x)\right|<\lambda_{i}, \text { for all } i=1,2 \text { and } x \in(a, b) .
$$

Moreover, let $\phi \in \mathcal{C}_{c}^{\infty}(-1,1)$ with $\operatorname{supp}(\phi) \subseteq(a, b)$ such that also

$$
\left|\sigma_{i}\left[u_{\lambda_{1}, \lambda_{2}}^{*}\right](x)+\phi^{(2-i)}(x)\right|<\lambda_{i} \text {, for all } i=1,2 \text { and } x \in(a, b) .
$$


Then,

$$
w^{*}:=u_{\lambda_{1}, \lambda_{2}}^{*}+\phi^{\prime \prime} \in \mathcal{B}_{\mathrm{TGV}_{\lambda_{1}, \lambda_{2}}}^{*}
$$

and therefore it follows from (5.24) that

$$
-\int_{a}^{b} \phi^{\prime \prime} u_{\lambda_{1}, \lambda_{2}} d x \leq 0, \quad \forall \phi \in \mathcal{C}_{c}^{\infty}(-1,1) \text { with } \operatorname{supp}(\phi) \subseteq(a, b) .
$$

Hence, $u_{\lambda_{1}, \lambda_{2}}$ is a polynomial of order one in the interval $(a, b)$.

Item 2:

- i) Assume that

$$
\sigma^{1}\left[u_{\lambda_{1}, \lambda_{2}}^{*}\right](x)=\lambda_{1}, \quad \forall x \in(a, b)
$$

Then

$$
u^{\delta}(x)-u_{\lambda_{1}, \lambda_{2}}(x)=u_{\lambda_{1}, \lambda_{2}}^{*}(x)=\left(\sigma^{1}\left[u_{\lambda_{1}, \lambda_{2}}^{*}\right]\right)^{\prime}(x)=0, \quad \forall x \in(a, b),
$$

and therefore $u^{\delta}(x)=u_{\lambda_{1}, \lambda_{2}}(x)$ in $(a, b)$.

ii) Assume that

$$
\left|\sigma^{2}\left[u_{\lambda_{1}, \lambda_{2}}^{*}\right](x)\right|=\lambda_{2}, \quad \forall x \in(a, b) .
$$

From this it follows that

$$
\begin{aligned}
0 & =\left(\sigma^{2}\left[u_{\lambda_{1}, \lambda_{2}}^{*}\right]\right)^{\prime \prime}(x) \\
& =\left(\sigma^{1}\left[u_{\lambda_{1}, \lambda_{2}}^{*}\right]\right)^{\prime}(x) \\
& =u_{\lambda_{1}, \lambda_{2}}(x)-u^{\delta}(x), \quad \forall x \in(a, b),
\end{aligned}
$$

and therefore $u^{\delta}(x)=u_{\lambda_{1}, \lambda_{2}}(x)$ in $(a, b)$.

Item 4: Item 4 is based on the assumption that there exists $\epsilon>0$ and $x_{0} \in(-1,1)$ such that

$$
\sigma^{2}\left[u_{\lambda_{1}, \lambda_{2}}^{*}\right]\left(x_{0}\right)=\lambda_{2} \text { and } \sigma^{2}\left[u_{\lambda_{1}, \lambda_{2}}^{*}\right]\left(x_{0} \pm y\right)<\lambda_{2}, \quad \forall y \in(0, \epsilon) .
$$

Then, from Item 1 it follows that $u_{\lambda_{1}, \lambda_{2}}$ is piecewise affine linear in $\left(x_{0}-\epsilon, x_{0}+\epsilon\right)$. To be precise, there exist coefficients $c_{1}, d_{1}, c_{2}, d_{2}$ such that

$$
u_{\lambda_{1}, \lambda_{2}}(x)= \begin{cases}c_{1} x+d_{1}, & \forall x \in\left(x_{0}-\epsilon, x_{0}\right), \\ c_{2} x+d_{2}, & \forall x \in\left(x_{0}, x_{0}+\epsilon\right) .\end{cases}
$$

We prove the assertion of Item 4 in two steps.

1. Firstly we show that the coefficients of the piecewise polynomial satisfy $c_{1} \geq$ $c_{2}$.

2. Secondly we show that $u_{\lambda_{1}, \lambda_{2}}$ is continuous at $x_{0}$, such that we can conclude that it is bending at $x_{0}$. 


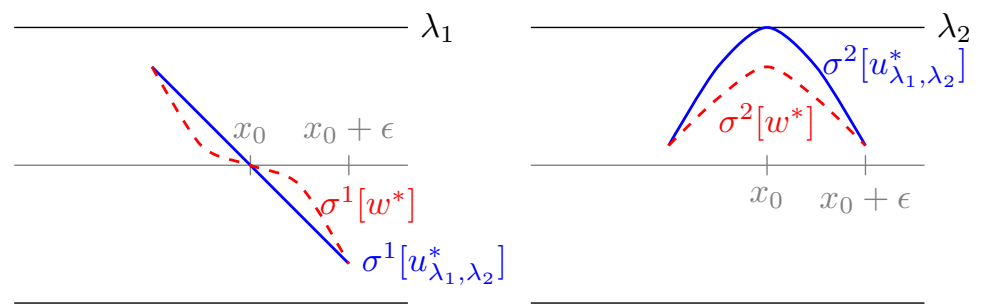

FIG. 5.2. The figure shows the construction of $w^{*}$ satisfying (5.27) and (5.28).

a) To prove the first item, $c_{1} \geq c_{2}$, we use some $w^{*} \in \mathcal{B}_{\mathrm{TGV}_{\lambda_{1}, \lambda_{2}}}^{*}$ (see figure 5.2) satisfying

$$
\begin{gathered}
\sigma^{1}\left[w^{*}\right]\left(x_{0}\right)=0, \\
\sigma^{1}\left[w^{*}\right](x)=\sigma^{1}\left[u_{\lambda_{1}, \lambda_{2}}^{*}\right](x), \quad \forall x \notin\left(x_{0}-\epsilon, x_{0}+\epsilon\right), \\
\sigma^{1}\left[w^{*}\right](x) \leq \sigma^{1}\left[u_{\lambda_{1}, \lambda_{2}}^{*}\right](x), \quad \forall x \in\left(x_{0}-\epsilon, x_{0}\right), \\
\sigma^{1}\left[w^{*}\right](x) \geq \sigma^{1}\left[u_{\lambda_{1}, \lambda_{2}}^{*}\right](x), \quad \forall x \in\left(x_{0}, x_{0}+\epsilon\right), \\
\mu:=-\int_{x_{0}-\epsilon}^{x_{0}}\left(\sigma^{1}\left[w^{*}\right]-\sigma^{1}\left[u_{\lambda_{1}, \lambda_{2}}^{*}\right]\right) d x \\
=\int_{x_{0}}^{x_{0}+\epsilon}\left(\sigma^{1}\left[w^{*}\right]-\sigma^{1}\left[u_{\lambda_{1}, \lambda_{2}}^{*}\right]\right) d x
\end{gathered}
$$

and

$$
\begin{aligned}
\sigma^{2}\left[w^{*}\right](x) & <\sigma^{2}\left[u_{\lambda_{1}, \lambda_{2}}^{*}\right](x), \quad \forall x \in\left(x_{0}-\epsilon, x_{0}+\epsilon\right) \backslash\left\{x_{0}\right\} \\
0 & <\sigma^{2}\left[u_{\lambda_{1}, \lambda_{2}}^{*}\right]\left(x_{0}\right)-\sigma^{2}\left[w^{*}\right]\left(x_{0}\right)<\lambda_{2} .
\end{aligned}
$$

With such a function $w^{*}$ it follows from (5.24) that

$$
\begin{aligned}
0 & \leq \int_{-1}^{1} u_{\lambda_{1}, \lambda_{2}}\left(w^{*}-u_{\lambda_{1}, \lambda_{2}}^{*}\right) d x \\
& =-\int_{x_{0}-\epsilon}^{x_{0}+\epsilon} u_{\lambda_{1}, \lambda_{2}}^{\prime}\left(\sigma^{1}\left[w^{*}\right]-\sigma^{1}\left[u_{\lambda_{1}, \lambda_{2}}^{*}\right]\right) d x \\
& =c_{1} \underbrace{\int_{x_{0}-\epsilon}^{x_{0}}\left(\sigma^{1}\left[w^{*}\right]-\sigma^{1}\left[u_{\lambda_{1}, \lambda_{2}}^{*}\right]\right) d x}_{=-\mu}+c_{2}^{\int_{x_{0}}^{x_{0}+\epsilon}\left(\sigma^{1}\left[w^{*}\right]-\sigma^{1}\left[u_{\lambda_{1}, \lambda_{2}}^{*}\right]\right) d x} \\
& =\mu\left(c_{2}-c_{1}\right),
\end{aligned}
$$

which shows that $c_{1} \leq c_{2}$ because $\mu \geq 0$.

b) To prove the continuity of $u_{\lambda_{1}, \lambda_{2}}$ we use a function $w^{*} \in \mathcal{B}_{\mathrm{TGV}_{\lambda_{1}, \lambda_{2}}}^{*}$ which satisfies

$$
\begin{array}{ll}
\sigma^{2}\left[w^{*}\right](x)=\sigma^{2}\left[u_{\lambda_{1}, \lambda_{2}}^{*}\right](x), & \forall x \notin\left(x_{0}-\epsilon, x_{0}+\epsilon\right), \\
\sigma^{2}\left[w^{*}\right](x) \neq \sigma^{2}\left[u_{\lambda_{1}, \lambda_{2}}^{*}\right](x), & \text { for a. a. } x \in\left(x_{0}-\epsilon, x_{0}+\epsilon\right),
\end{array}
$$


188 EXACT SOLUTION OF ONE-DIMENSIONAL TOTAL GENERALIZED VARIATION

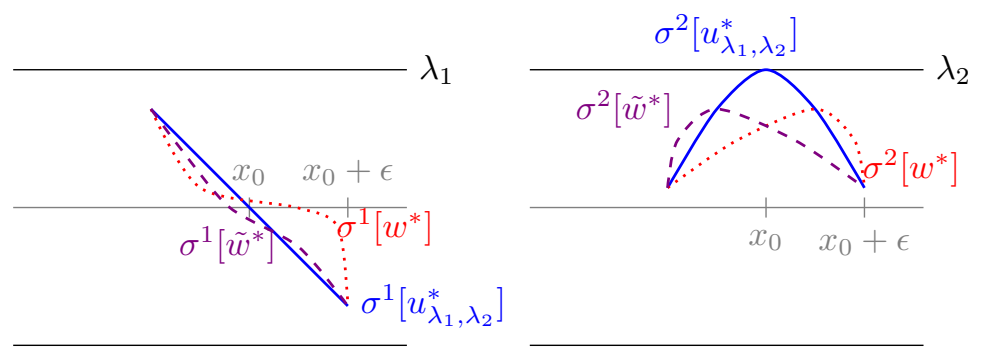

FIG. 5.3. $w^{*}$ satisfying (5.29).

$$
\begin{aligned}
\sigma^{1}\left[w^{*}-u^{*}\right]\left(x_{0}\right) & =a \neq 0 \\
\int_{x_{0}-\epsilon}^{x_{0}}\left(\sigma^{1}\left[w^{*}\right]-\sigma^{1}\left[u_{\lambda_{1}, \lambda_{2}}^{*}\right]\right) d x & =-\int_{x_{0}}^{x_{0}+\epsilon}\left(\sigma^{1}\left[w^{*}\right]-\sigma^{1}\left[u_{\lambda_{1}, \lambda_{2}}^{*}\right]\right) d x .
\end{aligned}
$$

Such a function is represented in figure 5.3. With such a function $w^{*}$ it follows from $(5.24)$, (5.25), and integration by parts that

$$
\begin{aligned}
0 \leq & \int_{-1}^{1} u_{\lambda_{1}, \lambda_{2}}\left(w^{*}-u_{\lambda_{1}, \lambda_{2}}^{*}\right) d x \\
= & \underbrace{\sigma^{1}\left[w^{*}-u_{\lambda_{1}, \lambda_{2}}^{*}\right]\left(x_{0}\right)}_{=a}\left(-c_{1} x_{0}-d_{1}+c_{2} x_{0}+d_{2}\right) \\
& -c_{1} \int_{x_{0}-\epsilon}^{x_{0}}\left(\sigma^{1}\left[w^{*}\right]-\sigma^{1}\left[u_{\lambda_{1}, \lambda_{2}}^{*}\right]\right) d x-c_{2} \int_{x_{0}}^{x_{0}+\epsilon}\left(\sigma^{1}\left[w^{*}\right]-\sigma^{1}\left[u_{\lambda_{1}, \lambda_{2}}^{*}\right]\right) d x .
\end{aligned}
$$

Choosing $\tilde{w}^{*}$ such that $\sigma^{1}\left[\tilde{w}^{*}-u_{\lambda_{1}, \lambda_{2}}^{*}\right]\left(x_{0}\right)=-a$, but otherwise satisfying the same properties as $w^{*}$, i.e. (5.29a) and (5.29b), we then obtain

$$
\begin{aligned}
0 \leq & \int_{-1}^{1} u_{\lambda_{1}, \lambda_{2}}\left(\tilde{w}^{*}-u_{\lambda_{1}, \lambda_{2}}^{*}\right) d x \\
= & \underbrace{\sigma^{1}\left[\tilde{w}^{*}-u_{\lambda_{1}, \lambda_{2}}^{*}\right]\left(x_{0}\right)}_{=-a}\left(-c_{1} x_{0}-d_{1}+c_{2} x_{0}+d_{2}\right) \\
& \quad-c_{1} \int_{x_{0}-\epsilon}^{x_{0}}\left(\sigma^{1}\left[\tilde{w}^{*}\right]-\sigma^{1}\left[u_{\lambda_{1}, \lambda_{2}}^{*}\right]\right) d x-c_{2} \int_{x_{0}}^{x_{0}+\epsilon}\left(\sigma^{1}\left[\tilde{w}^{*}\right]-\sigma^{1}\left[u_{\lambda_{1}, \lambda_{2}}^{*}\right]\right) d x .
\end{aligned}
$$

Combining (5.31) and (5.30) finally shows

$$
\begin{aligned}
a\left(-c_{1} x_{0}-d_{1}+c_{2} x_{0}+d_{2}\right) & \leq 0 \\
& \leq a\left(-c_{1} x_{0}-d_{1}+c_{2} x_{0}+d_{2}\right),
\end{aligned}
$$

and we conclude that $-c_{1} x_{0}-d_{1}+c_{2} x_{0}+d_{2}=0$, which shows that $u_{\lambda_{1}, \lambda_{2}}$ is continuous at $x_{0}$.

Item 3: Assume that $u_{\lambda_{1}, \lambda_{2}}$ is as in (5.23). In the case where

$$
\begin{aligned}
\sigma^{1}\left[u_{\lambda_{1}, \lambda_{2}}^{*}\right]\left(x_{0}\right) & =\lambda_{1} \text { and } \\
\sigma^{1}\left[u_{\lambda_{1}, \lambda_{2}}^{*}\right](y) & <\lambda_{1}, \quad \forall y \in\left(x_{0}-\epsilon, x_{0}+\epsilon\right), \backslash\left\{x_{0}\right\},
\end{aligned}
$$


we select some $w^{*} \in \mathcal{H}^{2}$ such that

$$
\begin{gathered}
u_{\lambda_{1}, \lambda_{2}}^{*}(x)=w^{*}(x), \quad \forall x \notin\left(x_{0}-\epsilon, x_{0}+\epsilon\right), \\
u_{\lambda_{1}, \lambda_{2}}^{*}(x)<w^{*}(x), \quad \forall x \in\left(x_{0}-\epsilon, x_{0}\right), \\
u_{\lambda_{1}, \lambda_{2}}^{*}(x)>w^{*}(x), \quad \forall x \in\left(x_{0}, x_{0}+\epsilon\right),
\end{gathered}
$$

and

$$
\sigma^{1}\left[u_{\lambda_{1}, \lambda_{2}}^{*}\right]\left(x_{0}\right)=\sigma^{1}\left[w^{*}\right]\left(x_{0}\right)
$$

Defining

$$
a:=\int_{x_{0}-\epsilon}^{x_{0}} \sigma^{1}\left[w^{*}-u_{\lambda_{1}, \lambda_{2}}^{*}\right] d x \underset{(5.34)}{=}-\int_{x_{0}}^{x_{0}+\epsilon} \sigma^{1}\left[w^{*}-u_{\lambda_{1}, \lambda_{2}}^{*}\right] d x
$$

(5.24) can be rewritten as

$$
\begin{aligned}
0 & \geq \int_{-1}^{1} u_{\lambda_{1}, \lambda_{2}}\left(w^{*}-u_{\lambda_{1}, \lambda_{2}}^{*}\right) d x \\
& =-\left(c_{1} x_{0}+d_{1}\right) \sigma^{1}\left[w^{*}-u^{*}\right]\left(x_{0}\right)-c_{1} a+\left(c_{2} x_{0}+d_{2}\right) \sigma^{1}\left[w^{*}-u^{*}\right]\left(x_{0}\right)+c_{2} a \\
& =a\left(c_{2}-c_{1}\right) .
\end{aligned}
$$

Now replacing conditions (5.32), (5.33), by

$$
\begin{array}{ll}
u_{\lambda_{1}, \lambda_{2}}^{*}(x)>w^{*}(x), & \forall x \in\left(x_{0}-\epsilon, x_{0}\right), \\
u_{\lambda_{1}, \lambda_{2}}^{*}(x)<w^{*}(x), & \forall x \in\left(x_{0}, x_{0}+\epsilon\right),
\end{array}
$$

and again using (5.24) we also obtain $a\left(c_{1}-c_{2}\right) \leq 0$. Thus $c_{1}=c_{2}$ (jumping down when $\left.\sigma^{1}\left[u_{\lambda_{1}, \lambda_{2}}^{*}\right]=+\lambda_{1}\right)$. Using the same arguments as in previous items, we can also show that $d_{1} \geq d_{2}$.

Lemma 5.7. Let $u_{\lambda_{1}, \lambda_{2}}$ be the minimizer of $u \rightarrow \mathcal{S}(u)+T G V_{\lambda_{1}, \lambda_{2}}(u)$.

1. If there exists $x_{0} \in(-1,1)$, such that $u_{\lambda_{1}, \lambda_{2}}$ is as in (5.21) (jumping up $d_{1} \leq$ $\left.d_{2}\right)$, then $\sigma^{1}\left[u_{\lambda_{1}, \lambda_{2}}^{*}\right]\left(x_{0}\right)=\lambda_{1}$.

2. If there exists $x_{0} \in(-1,1)$, such that $u_{\lambda_{1}, \lambda_{2}}$ is as in (5.23) and $c_{2} \leq c_{1}$ (negative bending), then $\sigma^{2}\left[u_{\lambda_{1}, \lambda_{2}}^{*}\right]\left(x_{0}\right)=\lambda_{2}$.

3. If there exists an interval $A$ such that $u_{\lambda_{1}, \lambda_{2}}(x)=u^{\delta}(x)$ for $x \in A$, then one of the following two statements holds:

(a) $\left|\sigma^{1}\left[u_{\lambda_{1}, \lambda_{2}}^{*}\right](x)\right|=\lambda_{1}$ for $x \in A$, or

(b) $\left|\sigma^{2}\left[u_{\lambda_{1}, \lambda_{2}}^{*}\right](x)\right|=\lambda_{2}$ and $\sigma^{1}\left[u_{\lambda_{1}, \lambda_{2}}^{*}\right](x)=0$ for $x \in A$.

Proof. Recall that if $u_{\lambda_{1}, \lambda_{2}}$ is different from $u^{\delta}$, then $u_{\lambda_{1}, \lambda_{2}}$ is a polynomial (piecewise). Set $I:=\left(x_{0}-\epsilon, x_{0}+\epsilon\right)$. 
1. Now assume that $u_{\lambda_{1}, \lambda_{2}}$ is as in (5.21) and $\sigma^{1}\left[u_{\lambda_{1}, \lambda_{2}}^{*}\right](x)<\lambda_{1}$ for $x \in I$. Then we can find $w^{*} \in \mathcal{B}_{1}^{*} \lambda_{1} \cap \mathcal{B}_{2}^{*} \lambda_{2}$ such that

$$
\begin{aligned}
w^{*}(x) & =u_{\lambda_{1}, \lambda_{2}}^{*}(x), & & \text { for } x \in(-1,1) \backslash I, \\
\sigma^{1}\left[w^{*}\right]\left(x_{0}\right) & >\sigma^{1}\left[u_{\lambda_{1}, \lambda_{2}}^{*}\right]\left(x_{0}\right), & & \\
\sigma^{2}\left[w^{*}\right](x) & =\sigma^{2}\left[u_{\lambda_{1}, \lambda_{2}}^{*}\right](x), & & \text { for } x \in(-1,1) \backslash I .
\end{aligned}
$$

The last condition implies that

$$
\int_{x_{0}-\epsilon}^{x_{0}+\epsilon} x\left(u_{\lambda_{1}, \lambda_{2}}^{*}-w^{*}\right) d x=0
$$

so that

$$
\begin{aligned}
\int_{-1}^{1} u_{\lambda_{1}, \lambda_{2}}\left(u_{\lambda_{1}, \lambda_{2}}^{*}-w^{*}\right) d x & =d_{1} \int_{x_{0}-\epsilon}^{x_{0}}\left(u_{\lambda_{1}, \lambda_{2}}^{*}-w^{*}\right) d x+d_{2} \int_{x_{0}}^{x_{0}+\epsilon}\left(u_{\lambda_{1}, \lambda_{2}}^{*}-w^{*}\right) d x \\
& =\underbrace{\left(d_{2}-d_{1}\right)}_{\geq 0} \underbrace{\left(\sigma^{1}\left[w^{*}\right]\left(x_{0}\right)-\sigma^{1}\left[u_{\lambda_{1}, \lambda_{2}}^{*}\right]\left(x_{0}\right)\right)}_{>0}>0 .
\end{aligned}
$$

Now this, together with (4.4), would give

$$
\operatorname{TGV}_{\lambda_{1}, \lambda_{2}}\left(u_{\lambda_{1}, \lambda_{2}}\right)=-\int_{-1}^{1} u_{\lambda_{1}, \lambda_{2}} u_{\lambda_{1}, \lambda_{2}}^{*} d x<-\int_{-1}^{1} u_{\lambda_{1}, \lambda_{2}} w^{*} d x
$$

which contradicts the definition of $\mathrm{TGV}_{\lambda_{1}, \lambda_{2}}$ as the supremum of such integrals. Hence $\sigma^{1}\left[u_{\lambda_{1}, \lambda_{2}}^{*}\right]$ must be maximal at $x_{0}$.

2. Set $I:=\left(x_{0}-\epsilon, x_{0}+\epsilon\right)$ and assume that $u_{\lambda_{1}, \lambda_{2}}$ is as in (5.23) and $\sigma^{2}\left[u_{\lambda_{1}, \lambda_{2}}^{*}\right](x)<\lambda_{2}$ for $x \in I$. Then we can find $w^{*} \in \mathcal{B}_{\mathrm{TGV}_{\lambda_{1}, \lambda_{2}}}^{*}$ such that

$$
\begin{aligned}
w^{*}(x) & =u_{\lambda_{1}, \lambda_{2}}^{*}(x), & & \text { for } x \in(-1,1) \backslash I, \\
\sigma^{2}\left[w^{*}\right]\left(x_{0}\right) & >\sigma^{2}\left[u_{\lambda_{1}, \lambda_{2}}^{*}\right]\left(x_{0}\right), & & \\
\sigma^{1}\left[w^{*}\right](x) & =\sigma^{1}\left[u_{\lambda_{1}, \lambda_{2}}^{*}\right](x), & & \text { for } x \in(-1,1) \backslash I .
\end{aligned}
$$

The last condition and the continuity of $u_{\lambda_{1}, \lambda_{2}}$ at $x_{0}$ imply that

$$
\begin{aligned}
& \int_{x_{0}-\epsilon}^{x_{0}+\epsilon} u_{\lambda_{1}, \lambda_{2}}\left(u_{\lambda_{1}, \lambda_{2}}^{*}-w^{*}\right) d x \\
= & -\int_{x_{0}-\epsilon}^{x_{0}+\epsilon} u_{\lambda_{1}, \lambda_{2}}^{\prime} \sigma^{1}\left[u_{\lambda_{1}, \lambda_{2}}^{*}-w^{*}\right] d x \\
= & -c_{1} \int_{x_{0}-\epsilon}^{x_{0}} \sigma^{1}\left[u_{\lambda_{1}, \lambda_{2}}^{*}-w^{*}\right] d x-c_{2} \int_{\epsilon}^{x_{0}+\epsilon} \sigma^{1}\left[u_{\lambda_{1}, \lambda_{2}}^{*}-w^{*}\right] d x
\end{aligned}
$$

such that

$$
\int_{-1}^{1} u_{\lambda_{1}, \lambda_{2}}\left(u_{\lambda_{1}, \lambda_{2}}^{*}-w^{*}\right) d x=\underbrace{\left(c_{2}-c_{1}\right)}_{\leq 0} \underbrace{\left(\sigma^{2}\left[u_{\lambda_{1}, \lambda_{2}}^{*}-w^{*}\right]\left(x_{0}\right)\right)}_{<0}>0 .
$$


Now this, together with (4.4), would give

$$
\operatorname{TGV}_{\lambda_{1}, \lambda_{2}}\left(u_{\lambda_{1}, \lambda_{2}}\right)=-\int_{-1}^{1} u_{\lambda_{1}, \lambda_{2}} u_{\lambda_{1}, \lambda_{2}}^{*} d x<-\int_{-1}^{1} u_{\lambda_{1}, \lambda_{2}} w^{*} d x
$$

which contradicts the definition of $\mathrm{TGV}_{\lambda_{1}, \lambda_{2}}$ as a supremum. Hence $\sigma^{1}\left[u_{\lambda_{1}, \lambda_{2}}^{*}\right]$ must be maximal at $x_{0}$.

3. The proof is analogous to (1), (2).

\section{Example 1}

In the following we calculate the specific minimizers of $\mathrm{TV}, \mathrm{TV}^{2}$, and $\mathrm{TGV}_{\lambda_{1}, \lambda_{2}}$ minimization for the test data

$$
\begin{aligned}
u^{\delta}:(-1,1) & \rightarrow \mathbb{R} \\
x & \mapsto|x|-\frac{1}{2} .
\end{aligned}
$$

In this case we have

$$
\lambda_{1}\left\|u^{\delta}\right\|_{*, \mathrm{TV}_{\lambda_{1}}^{1}}=\left\|u^{\delta}\right\|_{*, \mathrm{TV}_{1}^{1}}=\left\|\sigma^{1}\left[u^{\delta}\right]\right\|_{L^{\infty}}=\int_{-1}^{-\frac{1}{2}} u^{\delta} d x=\frac{1}{8}
$$

and

$$
\lambda_{2}\left\|u^{\delta}\right\|_{*, \mathrm{TV}_{\lambda_{2}}^{2}}=\left\|u^{\delta}\right\|_{*, \mathrm{TV}_{1}^{2}}=\left\|\sigma^{2}\left[u^{\delta}\right]\right\|_{L^{\infty}}=\left|\sigma^{2}\left[u^{\delta}\right](0)\right|=\frac{1}{12}=\frac{2}{3}\left\|u^{\delta}\right\|_{*, \mathrm{TV}_{1}^{1}} .
$$

6.1. TV-minimizer. Using the same methods as in [15], we find that for given data (6.1), the minimizer of the TV-functional $\mathcal{F}^{1}$ is given by

$$
v_{\lambda_{1}}^{1}= \begin{cases}\sqrt{2 \lambda_{1}}-\frac{1}{2}, & |x| \leq \sqrt{2 \lambda_{1}}, \\ u^{\delta}(x), & \sqrt{2 \lambda_{1}}<|x| \leq 1-\sqrt{2 \lambda_{1}}, \\ \frac{1}{2}-\sqrt{2 \lambda_{1}}, & 1-\sqrt{2 \lambda_{1}}<|x| \leq 1\end{cases}
$$

(see figure 6.3 (right)).

The function $v_{\lambda_{1}}^{1}$ and its dual $v_{\lambda_{1}}^{1 *}$ satisfy the following properties:

1. $\operatorname{TV}\left(v_{\lambda_{1}}^{1}\right)=2-4 \sqrt{2 \lambda_{1}}$,

2. $\left\|v_{\lambda_{1}}^{1 *}\right\|_{*, \mathrm{TV}_{1}^{1}}=\sigma^{1}\left[v_{\lambda_{1}}^{1 *}\right]\left(\frac{1}{2}\right)=\lambda_{1}$, and

3. $\left\|v_{\lambda_{1}}^{1 *}\right\|_{*, \mathrm{TV}_{1}^{2}}=\left|\sigma^{2}\left[v_{\lambda_{1}}^{1 *}\right](0)\right|=\lambda_{1}\left(1-\frac{2}{3} \sqrt{2 \lambda_{1}}\right)$.

Hence, from Lemma 5.3 it follows that, as long as

$$
\lambda_{2} \geq\left\|v_{\lambda_{1}}^{1 *}\right\|_{*, \mathrm{TV}_{1}^{2}}=\lambda_{1}\left(1-\frac{2}{3} \sqrt{2 \lambda_{1}}\right),
$$

the $\mathrm{TV}^{1}$-minimizer is also the $\mathrm{TGV}_{\lambda_{1}, \lambda_{2}}$-minimizer and

$$
\lambda_{1} \operatorname{TV}_{1}^{1}\left(v_{\lambda_{1}}^{1}\right)=\operatorname{TV}_{\lambda_{1}}^{1}\left(v_{\lambda_{1}}^{1}\right)=\operatorname{TGV}_{\lambda_{1}, \lambda_{2}}\left(v_{\lambda_{1}}^{1}\right)=\lambda_{1}\left(2-4 \sqrt{2 \lambda_{1}}\right) .
$$




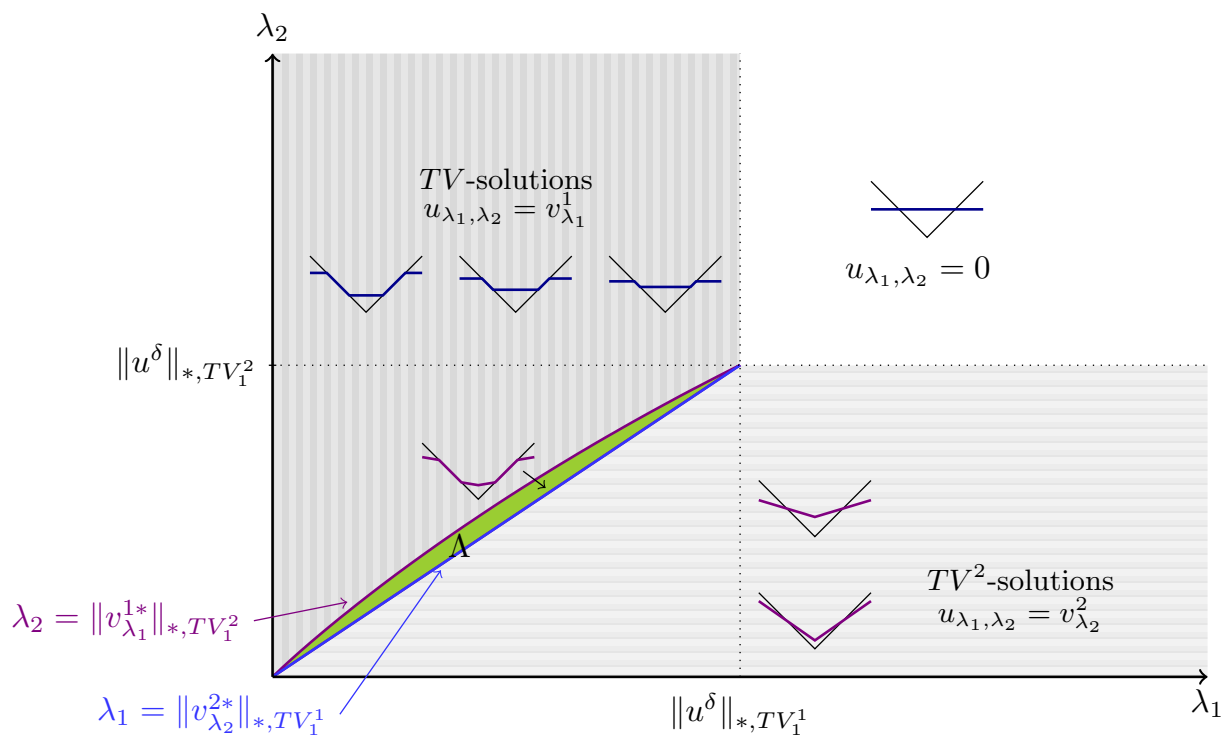

FIG. 6.1. This $\left(\lambda_{1}, \lambda_{2}\right)$-diagram shows regions where $\mathcal{G}$-minimizers are different or equal to $F^{i}$-minimizers. In the region with the horizontal lines we have $u_{\lambda_{1}, \lambda_{2}}=v_{\lambda_{2}}^{2}$, that is, the $T G V_{\lambda_{1}, \lambda_{2}}{ }^{-}$ minimizer equals the $T V^{2}$-minimizer. In the green region where $\left(\lambda_{1}, \lambda_{2}\right) \in \Lambda, T G V_{\lambda_{1}, \lambda_{2}}$-minimizers are different from $T V^{1}, T V^{2}$ minimizers, respectively.

6.2. $\mathbf{T V}^{2}$-minimizers. $\quad$ For $u^{\delta}$ from (6.1) the minimizer of $\mathcal{F}^{2}$ is given by

$$
v_{\lambda_{2}}^{2}=\left(1-\frac{1}{\left\|u^{\delta}\right\|_{*, \mathrm{TV}_{1}^{2}}} \lambda_{2}\right)^{+} u^{\delta}
$$

where

$$
f^{+}(x)=\max \{f(x), 0\} .
$$

Using Lemma 5.3 it follows that for

$$
\lambda_{1} \geq\left\|v_{\lambda_{2}}^{2 *}\right\|_{*, \mathrm{TV}_{1}^{1}}=\left(1-\frac{1}{\left\|u^{\delta}\right\|_{*, \mathrm{TV}_{1}^{2}}} \lambda_{2}\right)\left\|u^{\delta}\right\|_{*, \mathrm{TV}_{1}^{1}},
$$

the $\mathrm{TV}^{2}$-minimizer, i.e., the minimizer of $\mathcal{F}^{2}$, is also a minimizer of the $\mathrm{TGV}_{\lambda_{1}, \lambda_{2}}$ functional $\mathcal{G}$. In figure 6.1 we illustrate the $\left(\lambda_{1}, \lambda_{2}\right)$-region where the minimizers of $\mathcal{G}$ are equal to minimizers of $\mathcal{F}^{2}$.

6.3. $\mathbf{T G V} \mathbf{V}_{\lambda_{1}, \lambda_{2}}$-minimizer.

First, we calculate the set $\Lambda$ (cf. Definition 5.4) for which the $\mathrm{TGV}_{\lambda_{1}, \lambda_{2}}$-minimizer is different from the $\mathrm{TV}^{i}$-minimizers, respectively. For this particular data $u^{\delta}$ this means that for

$$
\lambda_{2} \notin \Lambda_{2}:=\left[\frac{1}{12}-\frac{2}{3} \lambda_{1},\left(1-\frac{2}{3} \sqrt{2 \lambda_{1}}\right) \lambda_{1}\right],
$$


the minimizer of the $\mathrm{TGV}_{\lambda_{1}, \lambda_{2}}$-functional $\mathcal{G}$ equals a minimizer of a $\mathrm{TV}^{i}$-functional $\mathcal{F}^{i}$, for some $i=1,2$.

Let now $\lambda_{2} \in \Lambda_{2}$, which is the only case for which we can expect that the $\operatorname{TGV}_{\lambda_{1}, \lambda_{2}}$ minimizer is different from $\mathrm{TV}^{i}$-minimizers.

We introduce the two-parametric set of functions $W$, consisting of all functions of the form

$$
w(x, c, d):= \begin{cases}d|x|+c(1-d)-\frac{1}{2}, & |x| \leq c, \\ u^{\delta}, & c<|x| \leq 1-c, \\ d|x|+c(d-1)-d+\frac{1}{2}, & |x|>1-c,\end{cases}
$$

where $c \in\left[0, \frac{1}{2}\right]$ and $d \in[0,1]$. Note that

- $w(x, c, d)$ is continuous,

- $w(x, 0,1)=w\left(x, \frac{1}{2}, 1\right)=u^{\delta}(x)$ and $w\left(x, \frac{1}{2}, 0\right)=0$,

- $w(x, c, 0)=v_{\lambda_{1}}^{1}(x)$ for $\lambda_{1}=\frac{1}{2} c^{2}$,

- $w\left(x, \frac{1}{2}, d\right)=v_{\lambda_{2}}^{2}(x)$ for $\lambda_{2}=(1-d)\left\|u^{\delta}\right\|_{*, \mathrm{TV}_{1}^{2}}$.

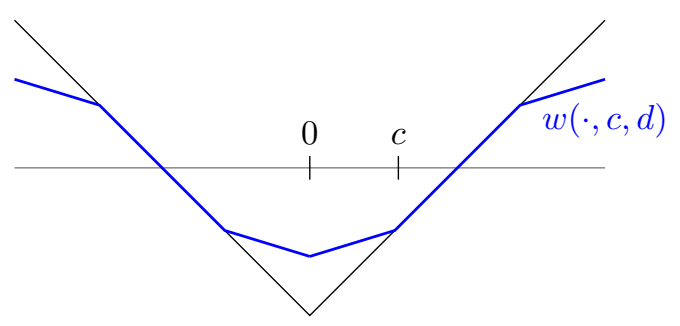

FiG. 6.2. Illustration of the function $w(\cdot, c, d)$.

Assuming that $w_{\lambda_{1}, \lambda_{2}}:=w\left(\cdot, c_{\lambda_{1}, \lambda_{2}}, d_{\lambda_{1}, \lambda_{2}}\right)$ minimizes $\mathcal{G}$, Lemma 5.7 provides necessary criteria for optimality of the parameters $c_{\lambda_{1}, \lambda_{2}}$ and $d_{\lambda_{1}, \lambda_{2}}$, which are derived in the following. Then, in Theorem 6.1 below, we prove that $w_{\lambda_{1}, \lambda_{2}}$ in fact minimizes $\mathcal{G}$.

Assuming that $w_{\lambda_{1}, \lambda_{2}}$ is a minimizer of $\mathcal{G}$ it follows from Lemma 5.7 that

- For $\left(\lambda_{1}, \lambda_{2}\right)$, such that $d_{\lambda_{1}, \lambda_{2}}>0, w_{\lambda_{1}, \lambda_{2}}$ bends at $x=0$. In Remark 6.1, we calculate the coefficients such that $d_{\lambda_{1}, \lambda_{2}}=0$. Item 2 of Lemma 5.7 states that then $w_{\lambda_{1}, \lambda_{2}}^{*}=w_{\lambda_{1}, \lambda_{2}}-u^{\delta}$ satisfies

$$
\sigma^{2}\left[w_{\lambda_{1}, \lambda_{2}}^{*}\right](0)=-\lambda_{2} .
$$

- Item 3a of Lemma 5.7 states that because $w_{\lambda_{1}, \lambda_{2}}(x)=u^{\delta}(x)$, $x \in\left(-1+c_{\lambda_{1}, \lambda_{2}},-c_{\lambda_{1}, \lambda_{2}}\right)$, we have

$$
\lambda_{1}=\left|\sigma^{1}\left[w_{\lambda_{1}, \lambda_{2}}^{*}\right]\left(c_{\lambda_{1}, \lambda_{2}}\right)\right|=\left|\sigma^{1}\left[w_{\lambda_{1}, \lambda_{2}}^{*}\right]\left(1-c_{\lambda_{1}, \lambda_{2}}\right)\right| .
$$

(Item $3 \mathrm{~b}$ cannot occur in this case, because $\sigma^{1}\left[w_{\lambda_{1}, \lambda_{2}}^{*}\right]\left(-1+c_{\lambda_{1}, \lambda_{2}}\right) \neq 0$, for any $d_{\lambda_{1}, \lambda_{2}} \neq 1$ )

Using a computer algebra system, we solve (6.5)-(6.6) and obtain

$$
c_{\lambda_{1}, \lambda_{2}}=\frac{3\left(\lambda_{1}-\lambda_{2}\right)}{2 \lambda_{1}}, \quad \quad d_{\lambda_{1}, \lambda_{2}}=1-\frac{8}{9} \frac{\lambda_{1}^{2}}{\left(\lambda_{2}-\lambda_{1}\right)^{2}} \lambda_{1} .
$$


REMARK 6.1. We want to see what happens for the special case when $\left(\lambda_{1}, \lambda_{2}\right) \in \partial \Lambda$, that is we consider the following two sets of parameters:

$$
\begin{aligned}
& \left\{\left(\lambda_{1},\left\|v_{\lambda_{1}}^{1 *}\right\|_{*, \mathrm{TV}_{1}^{2}}\right), \lambda_{1} \in\left(0,\left\|u^{\delta}\right\|_{*, \mathrm{TV}_{1}^{1}}\right)\right\}, \\
& \left\{\left(\left\|v_{\lambda_{2}}^{2 *}\right\|_{*, \mathrm{TV}_{1}^{1}}, \lambda_{2}\right), \lambda_{2} \in\left(0,\left\|u^{\delta}\right\|_{*, \mathrm{TV}_{1}^{2}}\right)\right\} .
\end{aligned}
$$

- In the case $\lambda_{2}=\lambda_{1}\left(1+\frac{2}{3} \sqrt{2 \lambda_{1}}\right)$ (this is the case where $\left\|v_{\lambda_{1}}^{1 *}\right\|_{*, \mathrm{TV}_{\lambda_{2}}^{2}}=1$; see Lemma 5.3), (6.7) gives $d_{\lambda_{1}, \lambda_{2}}=0$ and $c_{\lambda_{1}, \lambda_{2}}=\sqrt{2 \lambda_{1}}$. One can see that then $w_{\lambda_{1}, \lambda_{2}}$ is either piecewise constant or equal to $u^{\delta}$ on $\left(-1+c_{\lambda_{1}, \lambda_{2}},-c_{\lambda_{1}, \lambda_{2}}\right) \cup$ $\left(c_{\lambda_{1}, \lambda_{2}}, 1-c_{\lambda_{1}, \lambda_{2}}\right)$. We see that for this particular choice of $\left(\lambda_{1}, \lambda_{2}\right)$ we have $w_{\lambda_{1}, \lambda_{2}}=v_{\lambda_{1}}^{1}$, hence $w_{\lambda_{1}, \lambda_{2}}$ also minimizes $\mathcal{F}^{1}$.

- For $\lambda_{1}=\frac{3}{2} \lambda_{2}$ (this is the case where $\left\|v_{\lambda_{2}}^{2 *}\right\|_{*, \mathrm{TV}_{1}^{1}}=\lambda_{1}$; see Lemma 5.3), we have $c_{\lambda_{1}, \lambda_{2}}=\frac{1}{2}$ and $d_{\lambda_{1}, \lambda_{2}}=\left(1-\frac{\lambda_{2}}{\left\|u^{\delta}\right\|_{*, \mathrm{TV}_{1}^{2}}}\right)$. We see that $w_{\lambda_{1}, \lambda_{2}}=v_{\lambda_{2}}^{2}$.

Theorem 6.1. For $\left(\lambda_{1}, \lambda_{2}\right) \in \Lambda$ and $c_{\lambda_{1}, \lambda_{2}}, d_{\lambda_{1}, \lambda_{2}}$ satisfying (6.7), $u_{\lambda_{1}, \lambda_{2}}=w_{\lambda_{1}, \lambda_{2}}$.

In order to prove the theorem in a compact way, we need the following remark.

REMARK 6.2. In the next two items, we only rewrite $w_{\lambda_{1}, \lambda_{2}}$ as a linear combination of minimizers of $\mathcal{F}^{i}$, where we have to replace $\lambda_{i}$ by a different parameter $\mu_{i}$ depending on $\lambda_{1}, \lambda_{2}$.

- For given $\mu_{1} \in\left[0,\left\|u^{\delta}\right\|_{*, \mathrm{TV}_{1}^{1}}\right], \mu_{2} \in\left[0,\left\|u^{\delta}\right\|_{*, \mathrm{TV}_{1}^{2}}\right]$ set

$$
\lambda_{1}=\frac{\mu_{1} \mu_{2}}{\left\|u^{\delta}\right\|_{*, \mathrm{TV}_{1}^{2}}} \quad \text { and } \quad \lambda_{2}=\frac{\mu_{2}}{\left\|u^{\delta}\right\|_{*, \mathrm{TV}_{1}^{2}}}\left\|v_{\mu_{1}}^{1}\right\|_{*, \mathrm{TV}_{1}^{2}}=12 \mu_{2}\left(\mu_{1}-\frac{2}{3} \sqrt{2 \mu_{1}^{3}}\right) \text {. }
$$

Comparing the coefficients of the piecewise terms of $w_{\lambda_{1}, \lambda_{2}}$, we see that for $\left(\lambda_{1}, \lambda_{2}\right) \in \Lambda$ we can write

$$
w_{\lambda_{1}, \lambda_{2}}=\frac{\mu_{2}}{\left\|u^{\delta}\right\|_{*, \mathrm{TV}_{1}^{2}}} v_{\mu_{1}}^{1}+v_{\mu_{2}}^{2} .
$$

- On the other hand, for $\left(\lambda_{1}, \lambda_{2}\right) \in \Lambda$ given, we calculate $\mu_{1}, \mu_{2}$ by

$$
\mu_{2}=\frac{2}{27} \frac{\lambda_{1}^{2}}{\left(\lambda_{1}-\lambda_{2}\right)^{2}}, \quad \mu_{1}=\frac{9}{8} \frac{\left(\lambda_{1}-\lambda_{2}\right)^{2}}{\lambda_{1}^{2}}
$$

and express $w_{\lambda_{1}, \lambda_{2}}$ by (6.9).

Proof. Using the triangle-inequality and the estimate $\operatorname{TGV}_{\lambda_{1}, \lambda_{2}}(u) \leq \lambda_{1} T V^{i}(u)$, we obtain

$$
\begin{aligned}
\operatorname{TGV}_{\lambda_{1}, \lambda_{2}}\left(w_{\lambda_{1}, \lambda_{2}}\right) & \underset{(6.9)}{\leq} \operatorname{TGV}_{\lambda_{1}, \lambda_{2}}\left(\frac{\mu_{2}}{\left\|u^{\delta}\right\|_{*, \mathrm{TV}_{1}^{2}}} v_{\mu_{1}}^{1}\right)+\operatorname{TGV}_{\lambda_{1}, \lambda_{2}}\left(v_{\mu_{2}}^{2}\right) \\
& \leq \lambda_{1} \frac{\mu_{1}}{\left\|u^{\delta}\right\|_{*, \mathrm{TV}_{1}^{1}}} T V_{1}^{1}\left(v_{\mu_{1}}^{1}\right)+\lambda_{2} T V_{1}^{2}\left(v_{\mu_{2}}^{2}\right)
\end{aligned}
$$


Due to the definition of $\mathrm{TGV}_{\lambda_{1}, \lambda_{2}}$ and the choice of the parameters $\lambda_{1}, \lambda_{2}$, we have that $w_{\lambda_{1}, \lambda_{2}}^{*}:=w_{\lambda_{1}, \lambda_{2}}-u^{\delta} \in \mathcal{B}_{\mathrm{TGV}_{\lambda_{1}, \lambda_{2}}}^{*}$, such that

$$
-\int_{-1}^{1} w_{\lambda_{1}, \lambda_{2}}\left(w_{\lambda_{1}, \lambda_{2}}-u^{\delta}\right) d x \leq \operatorname{TGV}_{\lambda_{1}, \lambda_{2}}\left(w_{\lambda_{1}, \lambda_{2}}\right)
$$

In order to simplify the left side, we calculate

$$
\begin{aligned}
-\int_{-1}^{1} v_{\mu_{1}}^{1}\left(w_{\lambda_{1}, \lambda_{2}}-u^{\delta}\right) d x & =-\frac{\mu_{1}}{\left\|u^{\delta}\right\|_{*, \mathrm{TV}_{1}^{1}}} \int_{-1}^{1} v_{\mu_{1}}^{1}\left(v_{\mu_{1}}^{1}-u^{\delta}\right) d x \\
& =\frac{\mu_{1}}{\left\|u^{\delta}\right\|_{*, \mathrm{TV}_{1}^{1}}} \mu_{1} T V^{1}\left(v_{\mu_{1}}^{1}\right)
\end{aligned}
$$

and

$$
\begin{aligned}
& -\int_{-1}^{1} v_{\mu_{2}}^{2}\left(w_{\lambda_{1}, \lambda_{2}}-u^{\delta}\right) d x \\
& =-\left(1-\frac{\mu_{2}}{\left\|u^{\delta}\right\|_{*, \mathrm{TV}_{1}^{2}}}\right) \frac{\mu_{2}}{\left\|u^{\delta}\right\|_{*, \mathrm{TV}}^{2}} \int_{-1}^{1} u^{\delta}\left(v_{\mu_{1}}^{1}-u^{\delta}\right) d x \\
& =-\left(1-\frac{\mu_{2}}{\left\|u^{\delta}\right\|_{*, \mathrm{TV}_{1}^{2}}}\right) \frac{\mu_{2}}{\left\|u^{\delta}\right\|_{*, \mathrm{TV}_{1}^{2}}}(\int_{-1}^{1}|x|\left(v_{\mu_{1}}^{1}-u^{\delta}\right) d x-\frac{1}{2} \underbrace{\int_{-1}^{1}\left(v_{\mu_{1}}^{1}-u^{\delta}\right) d x}_{\sigma^{1}\left[v_{\mu}^{*}\right](1)=0}) \\
& =-\underbrace{\left(1-\frac{\mu_{2}}{\left\|u^{\delta}\right\|_{*, T V_{1}^{2}}}\right)}_{T V^{2}\left(v_{\mu_{2}}^{2}\right) \frac{1}{2}} \frac{\mu_{2}}{\left\|u^{\delta}\right\|_{*, \mathrm{TV}_{1}^{2}}} \underbrace{\left(\int_{-1}^{0} \sigma^{1}\left[v_{\mu_{1}}^{1 *}\right] d x-\int_{0}^{1} \sigma^{1}\left[v_{\mu_{1}}^{1 *}\right] d x\right)}_{2 \sigma^{2}\left[v_{\mu_{1}}^{1}{ }^{*}\right](0)=2\left\|v_{\mu_{1}}^{1}\right\|_{*, \mathrm{TV}_{1}^{2}}=\lambda_{2} \frac{\left\|u^{\delta}\right\|_{*, T V_{1}^{2}}}{\mu_{2}}} \\
& \underset{(6.8)}{=} \lambda_{2} \mathrm{TV}_{1}^{2}\left(v_{\mu_{2}}^{2}\right) \text {. }
\end{aligned}
$$

In total we obtain

$$
\begin{aligned}
& -\int_{-1}^{1} w_{\lambda_{1}, \lambda_{2}}\left(w_{\lambda_{1}, \lambda_{2}}-u^{\delta}\right) d x \\
= & \frac{\mu_{1}}{\left\|u^{\delta}\right\|_{*, \mathrm{TV}_{1}^{1}}}=\underbrace{\lambda_{1}}_{\frac{\mu_{2}}{\left\|u^{\delta}\right\|_{*, \mathrm{TV}_{1}^{2}}^{2}}} T V^{1}\left(v_{\mu_{1}}^{1}\right)+\lambda_{2} T V_{1}^{2}\left(v_{\mu_{2}}^{2}\right) .
\end{aligned}
$$

Comparing with (6.10) and (6.11) we have

$$
\operatorname{TGV}_{\lambda_{1}, \lambda_{2}}\left(w_{\lambda_{1}, \lambda_{2}}\right)=-\int w_{\lambda_{1}, \lambda_{2}}\left(w_{\lambda_{1}, \lambda_{2}}-u^{\delta}\right)
$$

which together with Lemma 5.7 implies that $w_{\lambda_{1}, \lambda_{2}}$ is a minimizer of $\mathcal{G}$. 


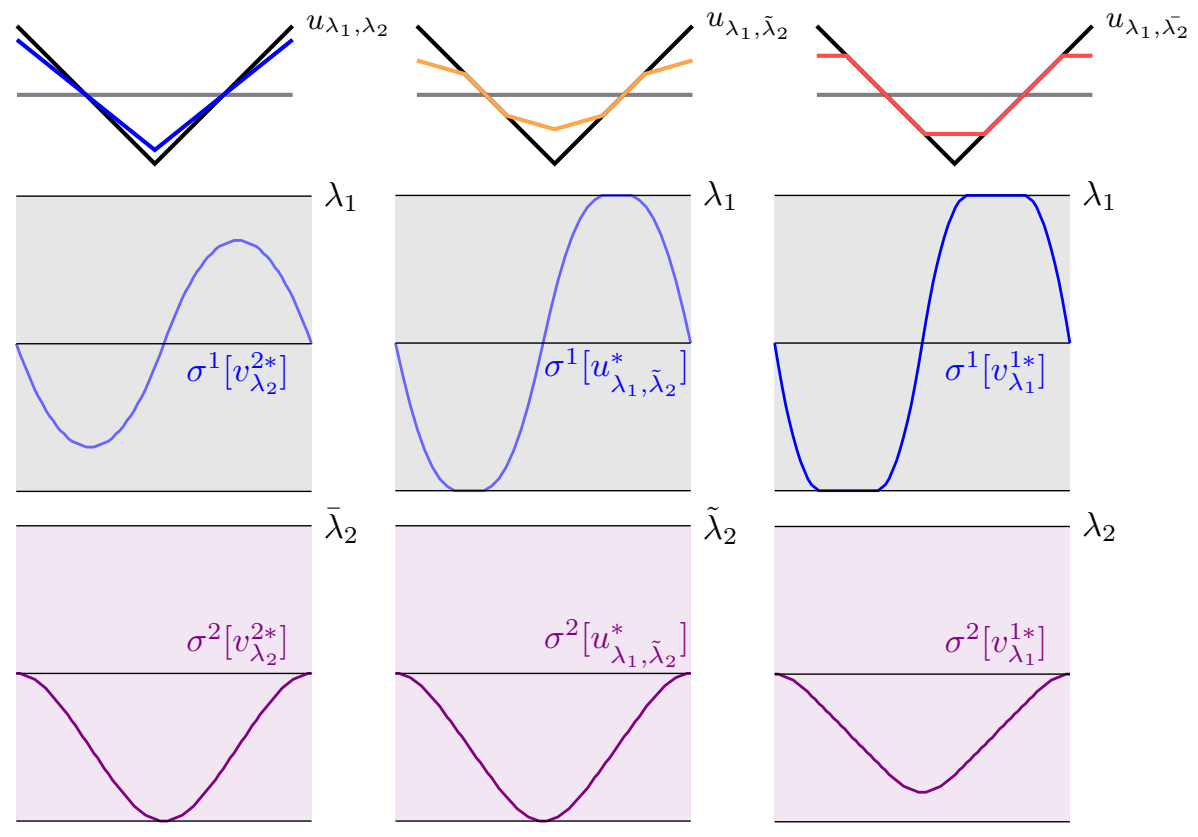

FIG. 6.3. $u_{\lambda_{1}, \lambda_{2}}$ for fixed $\lambda_{1}$ and changing $\lambda_{2}$. In this particular case we have $\tilde{\lambda}_{2} \in \Lambda_{2}$ and $\tilde{\lambda_{2}}, \lambda_{2} \notin \Lambda_{2}$ with $\lambda_{2}<\tilde{\lambda}_{2}<\overline{\lambda_{2}}$, such that $u_{\lambda_{1}, \bar{\lambda}_{2}}=v_{\lambda_{1}}^{1}$ and $u_{\lambda_{1}, \lambda_{2}}=v_{\lambda_{2}}^{2}$.

\section{Example 2}

Consider now as second test-data

$$
u^{\delta}(x)=1_{\left[-\frac{1}{2}, \frac{1}{2}\right]}(x)-\frac{1}{2},
$$

where $1_{[a, b]}$ is the indicator function of the interval $[a, b]$. Then

$$
\left\|u^{\delta}\right\|_{*, \mathrm{TV}_{1}^{1}}=\frac{1}{4}, \quad\left\|u^{\delta}\right\|_{*, \mathrm{TV}_{1}^{2}}=\frac{1}{8} .
$$

First we calculate minimizers of $\mathcal{F}^{i}$, as defined in (2.1), in order to obtain the sets $\left(\lambda_{1}, \lambda_{2}\right)$, where, according to Lemma 5.3 , the $\mathrm{TGV}_{\lambda_{1}, \lambda_{2}}$-minimizers are equal to some $\mathrm{TV}^{i}$-minimizers.

7.1. $\mathcal{F}^{1}$-minimizers. From [15], we know that $v_{\lambda_{1}}^{1}:=\left(1-\frac{\lambda_{1}}{\left\|u^{\delta}\right\|_{*, \mathrm{TV}}^{1}}\right) u^{\delta}$ minimizes $\mathcal{F}^{1}$ with the test data $u^{\delta}$. Furthermore, we have

$$
\left\|v_{\lambda_{1}}^{1 *}\right\|_{*, \mathrm{TV}_{1}^{2}}=\lambda_{1} \frac{\left\|u^{\delta}\right\|_{*, \mathrm{TV}_{1}^{2}}}{\left\|u^{\delta}\right\|_{*, \mathrm{TV}}^{1}}
$$

Applying Lemma 5.3, we conclude that $v_{\lambda_{1}}^{1}$ minimizes $\mathcal{G}$ as long as

$$
\lambda_{2} \geq\left\|v_{\lambda_{1}}-u^{\delta}\right\|_{*, \mathrm{TV}_{1}^{2}}=\lambda_{1} \frac{\left\|u^{\delta}\right\|_{*, \mathrm{TV}_{1}^{2}}}{\left\|u^{\delta}\right\|_{*, \mathrm{TV}_{1}^{1}}}=\frac{\lambda_{1}}{2}
$$




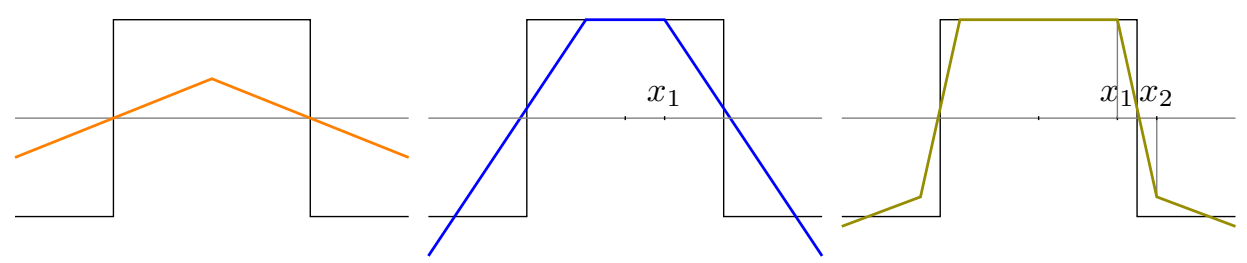

FIG. 7.1. Three different types of $\mathcal{F}^{2}$ minimizers $v_{\lambda_{2}}^{2}$ : They can bend once (3), twice (2) or four times (1).

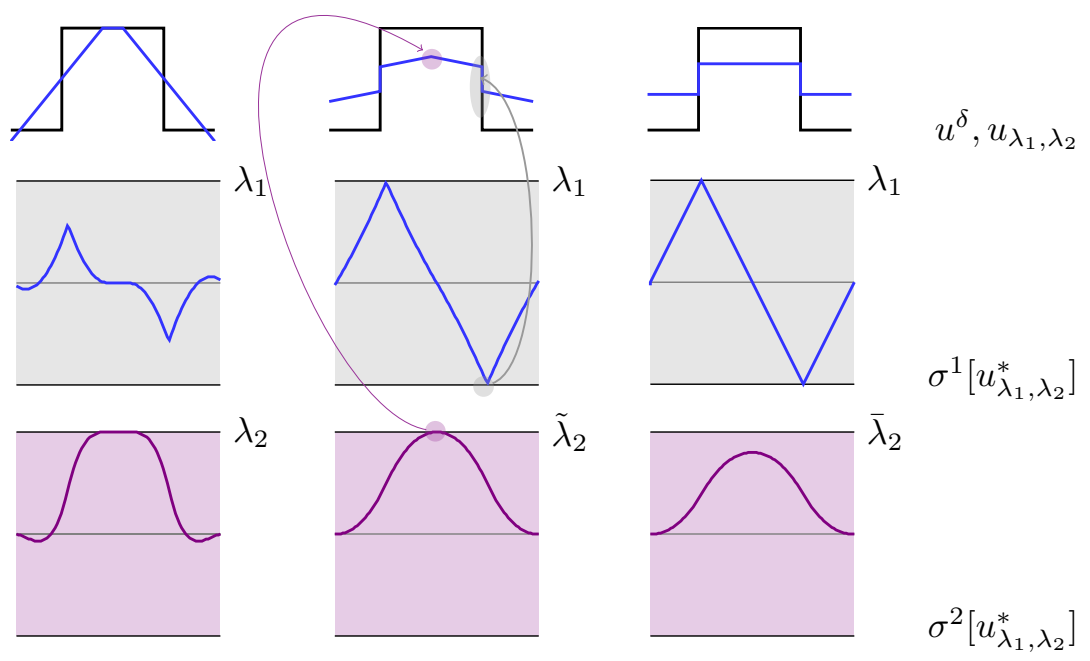

FIG. 7.2. $u_{\lambda_{1}, \lambda_{2}}$ for fixed $\lambda_{1}$ and changing $\lambda_{2}$. In this particular case we have $\left(\lambda_{1}, \tilde{\lambda}_{2}\right) \in \Lambda$ and $\left(\lambda_{1}, \tilde{\lambda_{2}}\right),\left(\lambda_{1}, \lambda_{2}\right) \notin \Lambda$ with $\lambda_{2}<\tilde{\lambda}_{2}<\overline{\lambda_{2}}$, such that $u_{\lambda_{1}, \bar{\lambda}_{2}}=v_{\lambda_{1}}^{1}$ and $u_{\lambda_{1}, \lambda_{2}}=v_{\lambda_{2}}^{2}$.

7.2. $\mathcal{F}^{2}$-minimizers. There are 3 different types of solutions (see [15] and figure 7.1):

1. For $\lambda_{2} \in\left[0, \frac{1}{24}(\sqrt{2} \sqrt[4]{3}-\sqrt{3})\right) v_{\lambda_{2}}^{2}$ is bending four times and $u_{\lambda_{2}}=u^{\delta}$ in a region near $x=0$. $\left\|v_{\lambda_{2}}^{2 *}\right\|_{*, \mathrm{TV}_{1}^{1}}=\left\|\sigma^{1}\left[v_{\lambda_{2}}^{2 *}\right]\right\|_{L^{\infty}}=g\left(\lambda_{2}\right)$, where $g\left(\lambda_{2}\right)$ is a ratio of polynomials of higher order in $\lambda_{2}$, not written explicitly here.

2. For $\lambda_{2} \in\left(\frac{1}{24}(\sqrt{2} \sqrt[4]{3}-\sqrt{3}), \frac{1}{24}\right) v_{\lambda_{2}}^{2}$ bends at $x_{1}= \pm 6 \lambda_{2}-\frac{1}{4}$, and $v_{\lambda_{2}}^{2}=u^{\delta}$ in a region near 0. Moreover $\left\|v_{\lambda_{2}}^{2}\right\|_{*, \mathrm{TV}_{1}^{1}}=\frac{1}{18} \frac{1+48 \lambda_{2}+576 \lambda_{2}{ }^{2}}{\left(1+8 \lambda_{2}\right)^{2}}$.

3. For $\lambda_{2} \in\left(\frac{1}{24}, \frac{1}{8}\right) v_{\lambda_{2}}^{2}$ is bending once and $v_{\lambda_{2}}^{2}(x)=\left(\frac{3}{2}-12 \lambda_{2}\right)\left(\frac{1}{2}-|x|\right)$. Additionally we can calculate $\left\|v_{\lambda_{2}}^{2 *}\right\|_{*, \mathrm{TV}_{1}^{1}}=\frac{1}{16}+\frac{3}{2} \lambda_{2}$.

The expressions $\left\|v_{\lambda_{2}}^{2}-u^{\delta}\right\|_{*, \mathrm{TV}_{1}^{1}}$ are used to calculate $\Lambda$, the set of parameters, where the $\mathcal{G}$-minimizer might be different to the $\mathcal{F}^{1}$ or $\mathcal{F}^{2}$-minimizer. 


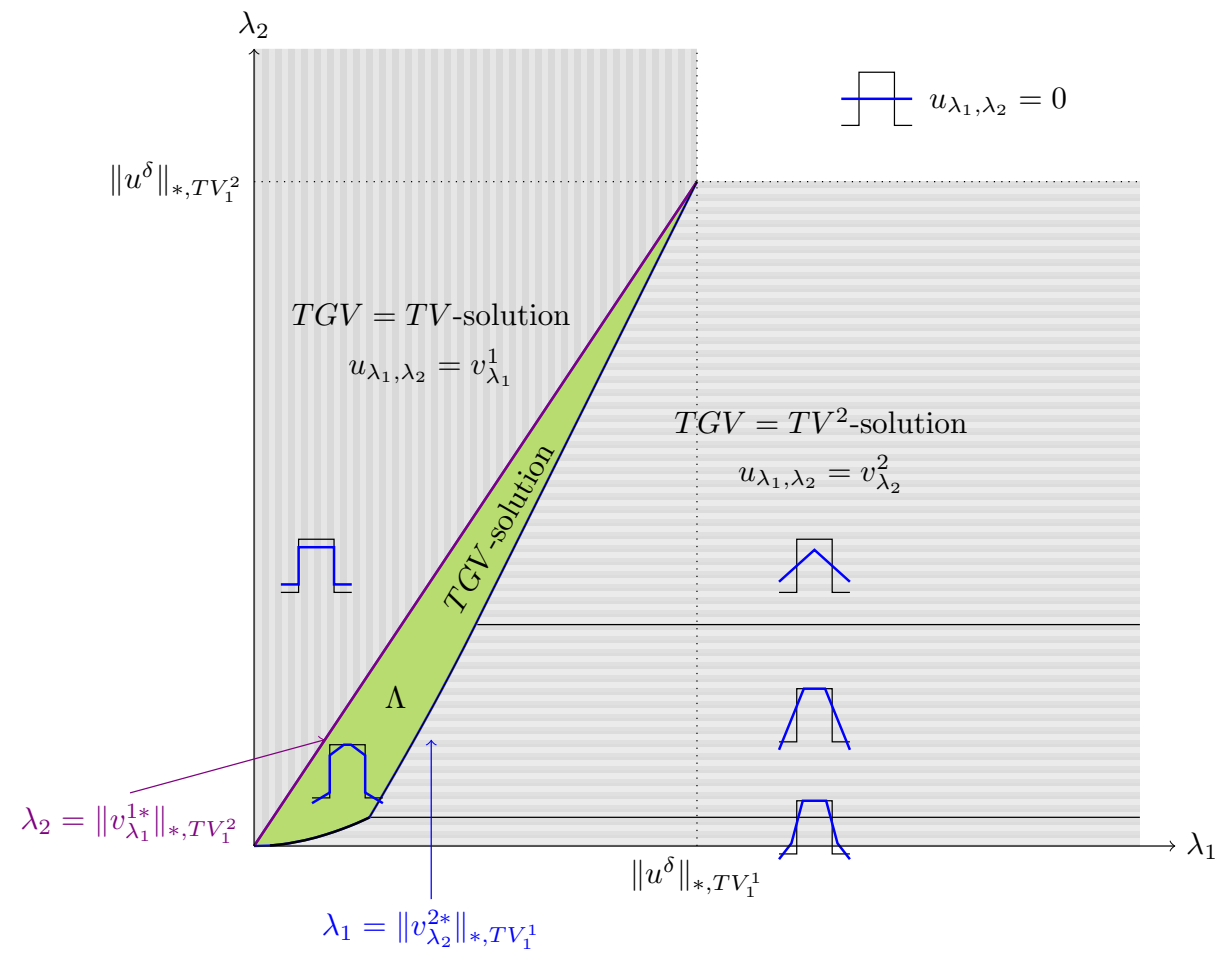

FIG. 7.3. Solid green region: $\Lambda$, here $u_{\lambda_{1}, \lambda_{2}} \neq v_{\lambda_{i}}^{i}$. Gray region: $u_{\lambda_{1}, \lambda_{2}}=v_{\lambda_{i}}^{i}$, vertical lines: $i=1$, horizontal lines: $i=2$.

We write the solutions in the form

$$
v_{\lambda_{2}}^{2}= \begin{cases}u^{\delta}(x), & \text { for }|x|<x_{1}, \\ k_{1}|x|+d_{1}, & \text { for } x_{1} \leq|x|<x_{2}, \\ k_{2}|x|+d_{2}, & \text { for } x_{2} \leq|x| \leq 1\end{cases}
$$

keeping in mind that $x_{1}$ can be 0 (third case), or $x_{2}$ can be larger than one (third and second case). $v_{\lambda_{1}}^{1}$ bends at $x_{i}$, such that $\sigma^{2}\left[v_{\lambda_{1}}^{1 *}\right]$ is extremal at $x_{0}$ (hence $\pm \lambda_{2}$ ). Thus, the coefficients $d_{i}, k_{i}$ are determined by the following equations:

$$
\begin{array}{lll}
\sigma^{2}\left[v_{\lambda_{1}}^{1 *}\right]\left(x_{1}\right)=\lambda_{2}, \sigma^{1}\left[v_{\lambda_{1}}^{1 *}\right]\left(x_{1}\right)=0, & & \text { cases } 1,2,3, \\
\sigma^{2}\left[v_{\lambda_{1}}^{1 *}\right]\left(x_{2}\right)=-\lambda_{2}, \sigma^{1}\left[v_{\lambda_{1}}^{1 *}\right]\left(x_{2}\right)=0, & & \text { case } 1 .
\end{array}
$$

7.3. $\mathcal{G}$-minimizers. We consider the same approach as for the previous example. Hence, first we calculate the set $\Lambda$ as in Definition 5.4, which is illustrated as the green (solid) set in figure 7.3. We have

$$
\begin{aligned}
\partial \Lambda=\{ & \left.\left(\lambda_{1},\left\|v_{\lambda_{1}}^{1 *}\right\|_{*, \mathrm{TV}_{1}^{2}}\right): \lambda_{1} \in\left[0,\left\|u^{\delta}\right\|_{*, \mathrm{TV}_{1}^{1}}\right]\right\} \\
& \cup\left\{\left(\left\|v_{\lambda_{2}}^{2 *}\right\|_{*, \mathrm{TV}_{1}^{1}}, \lambda_{2}\right): \lambda_{2} \in\left[0,\left\|u^{\delta}\right\|_{*, \mathrm{TV}_{1}^{2}}\right]\right\} .
\end{aligned}
$$

Next we set up a general ansatz function $w_{\lambda_{1}, \lambda_{2}}$ of piecewise affine functions, that bends once, twice, or four times, and jumps at $x= \pm 0$.5. Setting $w_{\lambda_{1}, \lambda_{2}}^{*}=w_{\lambda_{1}, \lambda_{2}}-$ 
$u^{\delta}$, we find the coefficients (of the piecewise affine functions) by solving a number of non-linear equations coming from the condition $\left|\sigma^{1}\left[w_{\lambda_{1}, \lambda_{2}}^{*}\right](x)\right|=\lambda_{1}$ whenever the ansatz function jumps and $\left|\sigma^{2}\left[w_{\lambda_{1}, \lambda_{2}}^{*}\right](x)\right|=\lambda_{2}, \sigma^{1}\left[w_{\lambda_{1}, \lambda_{2}}^{*}\right](x)=0$ whenever the ansatz function bends. We omit the explicit formulas and further calculations.

Then for $\left(\lambda_{1}, \lambda_{2}\right) \in \Lambda$ given, we found that the minimizers of $\mathcal{G}$ can be written in a compact form as follows.

Theorem 7.1. Let $\left(\lambda_{1}, \lambda_{2}\right) \in \Lambda$ and $\mu_{1}, \mu_{2}$ be such that

$$
\lambda_{2}=\frac{\mu_{1} \mu_{2}}{\left\|u^{\delta}\right\|_{*, T V_{1}^{1}}}, \quad \quad \lambda_{1}=\frac{\mu_{1}}{\left\|u^{\delta}\right\|_{*, T V_{1}^{1}}}\left\|v_{\mu_{2}}^{2 *}\right\|_{*, T V_{1}^{1}} .
$$

Then for $u^{\delta}$ as in (7.1),

$$
u_{\lambda_{1}, \lambda_{2}}=v_{\mu_{1}}^{1}+\frac{\mu_{1}}{\left\|u^{\delta}\right\|_{*, T V_{1}^{1}}} v_{\mu_{2}}^{2}
$$

minimize $\mathcal{G}$.

Proof. Lemma 4.1 states that $u_{\lambda_{1}, \lambda_{2}}$ is a minimizer if $\operatorname{TGV}_{\lambda_{1}, \lambda_{2}}\left(u_{\lambda_{1}, \lambda_{2}}\right)=-\int_{-1}^{1} u_{\lambda_{1}, \lambda_{2}}\left(u_{\lambda_{1}, \lambda_{2}}-u^{\delta}\right) d x\left(\right.$ see (4.4)) and $u_{\lambda_{1}, \lambda_{2}}^{*} \in \mathcal{B}_{\mathrm{TGV}_{\lambda_{1}, \lambda_{2}}^{*}}^{*}$. Using (7.4) we can estimate $\mathrm{TGV}_{\lambda_{1}, \lambda_{2}}\left(u_{\lambda_{1}, \lambda_{2}}\right)$ by

$$
\begin{aligned}
\operatorname{TGV}_{\lambda_{1}, \lambda_{2}}\left(u_{\lambda_{1}, \lambda_{2}}\right) & \leq \operatorname{TGV}_{\lambda_{1}, \lambda_{2}}\left(v_{\mu_{1}}^{1}\right)+\frac{\mu_{1}}{\left\|u^{\delta}\right\|_{*, \mathrm{TV}_{1}^{1}}} \operatorname{TGV}_{\lambda_{1}, \lambda_{2}}\left(v_{\mu_{2}}^{2}\right) \\
& \leq \operatorname{TV}_{\lambda_{1}}^{1}\left(v_{\mu_{1}}^{1}\right)+\frac{\mu_{1}}{\left\|u^{\delta}\right\|_{*, \mathrm{TV}_{1}^{1}}} \operatorname{TV}_{\lambda_{2}}^{2}\left(v_{\mu_{2}}^{2}\right) .
\end{aligned}
$$

Because $v_{\mu_{1}}^{1}=\left(1-\frac{\mu_{1}}{\left\|u^{\delta}\right\|_{*, \mathrm{TV}}^{1}}\right) u^{\delta}$, we have

$$
u_{\lambda_{1}, \lambda_{2}}^{*}=\frac{\mu_{1}}{\left\|u^{\delta}\right\|_{*, \mathrm{TV}_{1}^{1}}} v_{\mu_{2}}^{2 *} .
$$

Note that from the choice of the parameters $\mu_{1}, \mu_{2}$ we have

$$
\begin{aligned}
\lambda_{1} & =\lambda_{1}\left\|u_{\lambda_{1}, \lambda_{2}}^{*}\right\|_{*, \mathrm{TV}_{\lambda_{1}}^{1}} \\
& =\left\|\sigma^{1}\left[v_{\mu_{1}}^{1}+\frac{\mu_{1}}{\left\|u^{\delta}\right\|_{*, \mathrm{TV}}^{1}} v_{\mu_{2}}^{2}-u^{\delta}\right]\right\|_{L^{\infty}} \\
& =\frac{\mu_{1}}{\left\|u^{\delta}\right\|_{*, \mathrm{TV}_{1}^{1}}}\left\|\sigma^{1}\left[v_{\mu_{2}}^{2}-u^{\delta}\right]\right\|_{L^{\infty}} \\
& =\frac{\mu_{1}}{\left\|u^{\delta}\right\|_{*, \mathrm{TV}_{1}^{1}}} \lambda_{1}\left\|v_{\mu_{2}}^{2 *}\right\|_{*, \mathrm{TV}_{\lambda_{1}}^{1}},
\end{aligned}
$$

and also

$$
\begin{aligned}
\lambda_{2} & =\lambda_{2}\left\|u_{\lambda_{1}, \lambda_{2}}^{*}\right\|_{*, \mathrm{TV}_{\lambda_{2}}^{2}} \\
& =\frac{\mu_{1}}{\left\|u^{\delta}\right\|_{*, \mathrm{TV}}}\left\|\sigma^{2}\left[v_{\mu_{2}}^{2}-u^{\delta}\right]\right\|_{L^{\infty}} \\
& =\frac{\mu_{1}}{\left\|u^{\delta}\right\|_{*, \mathrm{TV}_{1}^{1}}} \underbrace{\lambda_{2}\left\|v_{\mu_{2}}^{2 *}\right\|_{*, \mathrm{TV}_{\lambda_{2}}^{2}}}_{\mu_{2}}
\end{aligned}
$$


such that $u_{\lambda_{1}, \lambda_{2}}^{*} \in \mathcal{B}_{\mathrm{TGV}_{\lambda_{1}, \lambda_{2}}}^{*}$ and

$$
-\int_{-1}^{1} u_{\lambda_{1}, \lambda_{2}}\left(u_{\lambda_{1}, \lambda_{2}}-u^{\delta}\right) d x \leq \operatorname{TGV}_{\lambda_{1}, \lambda_{2}}\left(u_{\lambda_{1}, \lambda_{2}}\right)
$$

Moreover, we can write

$$
\begin{aligned}
& \int_{-1}^{1} u_{\lambda_{1}, \lambda_{2}}\left(u_{\lambda_{1}, \lambda_{2}}-u^{\delta}\right) d x \\
= & \int_{-1}^{1} v_{\mu_{1}}^{1}\left(u_{\lambda_{1}, \lambda_{2}}-u^{\delta}\right) d x+\frac{\mu_{1}}{\left\|u^{\delta}\right\|_{*, \mathrm{TV}_{1}^{1}}} \int_{-1}^{1} v_{\mu_{2}}^{2}\left(u_{\lambda_{1}, \lambda_{2}}-u^{\delta}\right) d x .
\end{aligned}
$$

Because $v_{\mu_{1}}^{1}=\left(1-\frac{\mu_{1}}{\left\|u^{\delta}\right\|_{*, \mathrm{TV}_{1}^{1}}}\right) u^{\delta}$ and $u^{\delta}(x) \in\left\{-\frac{1}{2}, \frac{1}{2}\right\}$, we obtain

$$
\begin{aligned}
& \int_{-1}^{1} v_{\mu_{1}}^{1}\left(u_{\lambda_{1}, \lambda_{2}}-u^{\delta}\right) d x \\
= & \frac{1}{2}\left(1-\frac{\mu_{1}}{\left\|u^{\delta}\right\|_{*, \mathrm{TV} 1}}\right) \\
& \left(-\int_{-1}^{-\frac{1}{2}}\left(u_{\lambda_{1}, \lambda_{2}}-u^{\delta}\right) d x+\int_{-\frac{1}{2}}^{\frac{1}{2}}\left(u_{\lambda_{1}, \lambda_{2}}-u^{\delta}\right) d x-\int_{\frac{1}{2}}^{1}\left(u_{\lambda_{1}, \lambda_{2}}-u^{\delta}\right) d x\right) \\
= & \frac{1}{2}\left(1-\frac{\mu_{1}}{\left\|u^{\delta}\right\|_{*, \mathrm{TV}_{1}^{1}}}\right)\left(-\sigma^{1}\left[u_{\lambda_{1}, \lambda_{2}}^{*}\right]\left(-\frac{1}{2}\right)+\sigma^{1}\left[u_{\lambda_{1}, \lambda_{2}}^{*}\right]\left(\frac{1}{2}\right)-\sigma^{1}\left[u_{\lambda_{1}, \lambda_{2}}^{*}\right]\left(-\frac{1}{2}\right)\right. \\
& \left.-\sigma^{1}\left[u_{\lambda_{1}, \lambda_{2}}^{*}\right](1)+\sigma^{1}\left[u_{\lambda_{1}, \lambda_{2}}^{*}\right]\left(\frac{1}{2}\right)\right) .
\end{aligned}
$$

Now by the choice of the parameter $\lambda_{1}$ we have $\sigma^{1}\left[u_{\lambda_{1}, \lambda_{2}}^{*}\right]\left(-\frac{1}{2}\right)=+\lambda_{1}$ and $\sigma^{1}\left[u_{\lambda_{1}, \lambda_{2}}^{*}\right]\left(\frac{1}{2}\right)=-\lambda_{1}$, so that the equation above simplifies to

$$
-\int_{-1}^{1} v_{\mu_{1}}^{1}\left(u_{\lambda_{1}, \lambda_{2}}-u^{\delta}\right) d x=\lambda_{1} 2\left(1-\frac{\mu_{1}}{\left\|u^{\delta}\right\|_{*, \mathrm{TV}_{1}^{1}}}\right)=\lambda_{1} T V^{1}\left(v_{\mu_{1}}^{1}\right) .
$$

Next it remains to calculate $\int_{-1}^{1} v_{\mu_{2}}^{2}\left(u_{\lambda_{1}, \lambda_{2}}-u^{\delta}\right) d x$. Because

$$
u_{\lambda_{1}, \lambda_{2}}-u^{\delta}=v_{\mu_{1}}^{1}-\frac{\mu_{1}}{\left\|u^{\delta}\right\|_{*, \mathrm{TV}_{1}^{1}}} v_{\mu_{2}}^{2}-u^{\delta}=\frac{\mu_{1}}{\left\|u^{\delta}\right\|_{*, \mathrm{TV}_{1}^{1}}}\left(v_{\mu_{2}}^{2}-u^{\delta}\right)
$$

we have

$$
\begin{aligned}
\int_{-1}^{1} v_{\mu_{2}}^{2}\left(u_{\lambda_{1}, \lambda_{2}}-u^{\delta}\right) d x & =\frac{\mu_{1}}{\left\|u^{\delta}\right\|_{*, \mathrm{TV}_{1}^{1}}} \int_{-1}^{1} v_{\mu_{2}}^{2}\left(v_{\mu_{2}}^{2}-u^{\delta}\right) d x \\
& =-\frac{\mu_{1}}{\left\|u^{\delta}\right\|_{*, \mathrm{TV}_{1}^{1}}} \mu_{2} T V_{1}^{2}\left(v_{\mu_{2}}^{2}\right)
\end{aligned}
$$

where we used $T V_{1}^{2}\left(v_{\mu_{2}}^{2}\right)=-\mu_{2} \int_{-1}^{1} v_{\mu_{2}}^{2}\left(v_{\mu_{2}}^{2}-u^{\delta}\right) d x$, the optimality condition for $\mathcal{F}^{2}$ minimizers as in (4.3). Hence in total, using the connections between $\lambda_{i}$ and $\mu_{i}$, we obtain

$$
-\int_{-1}^{1} u_{\lambda_{1}, \lambda_{2}}\left(u_{\lambda_{1}, \lambda_{2}}-u^{\delta}\right) d x=\lambda_{1} \mathrm{TV}_{1}^{1}\left(v_{\mu_{1}}^{1}\right)+\lambda_{2} \frac{\mu_{1}}{\left\|u^{\delta}\right\|_{*, \mathrm{TV}_{1}^{1}}} \operatorname{TV}_{1}^{2}\left(v_{\mu_{2}}^{2}\right)
$$


A comparison with (7.5) shows that $\operatorname{TGV}_{\lambda_{1}, \lambda_{2}}\left(u_{\lambda_{1}, \lambda_{2}}\right)=-\int_{-1}^{1} u_{\lambda_{1}, \lambda_{2}}\left(u_{\lambda_{1}, \lambda_{2}}-u^{\delta}\right) d x$, hence according to Lemma $4.1 u_{\lambda_{1}, \lambda_{2}}$ minimizes $\mathcal{G}$.

\section{Example III}

Finally we consider $u^{\delta}=x^{2}-\frac{1}{3}$ but only sketch the different minimizers of $\mathcal{F}_{i}$ and $\mathcal{G}$ in order to show that in general, minimizers of $\mathcal{G}$ cannot be written as a sum of $\mathcal{F}_{i^{-}}$ minimizers. We have $\left\|u^{\delta}\right\|_{*, \mathrm{TV}_{1}^{2}}=\frac{1}{12}=0.833,\left\|u^{\delta}\right\|_{*, \mathrm{TV}_{1}^{1}}=\frac{2 \sqrt{3}}{27} \sim 0.12$.

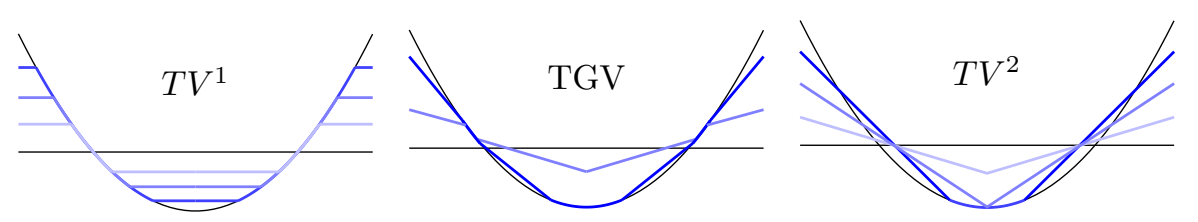

FIG. 8.1. Minimizers of $\mathcal{F}_{1}$ (left), $\mathcal{G}$ (middle), $\mathcal{F}_{2}$ (right), for different parameters.

8.1. $\mathcal{F}^{1}$-minimizers. Because $u^{\delta}$ is continuous, also the $\mathcal{F}_{1}$-minimizer is continuous. From the characterization of $\mathcal{F}_{1}$ minimizers we know that $v_{\lambda_{1}}^{1}$ is either equal to $u^{\delta}$ in an interval $\left( \pm c_{1}, \pm c_{2}\right)$ or constant $u^{\delta}\left(c_{1}\right), u^{\delta}\left(c_{2}\right)$ in the other intervals. In figure 8.1 (left), we illustrate $v_{\lambda_{1}}^{1}$ for different values of $\lambda_{1}$.

8.2. $\mathcal{F}^{2}$-minimizers. In this case, we have to consider two different types of minimizers.

- $\lambda_{2}$ large: (that is $\lambda \sim\left\|u^{\delta}\right\|_{*, \mathrm{TV}_{1}^{2}}$ ), $v_{\lambda_{2}}^{2}$ is piecewise constant and bends at $x=0$. Such solutions are constructed by considering the ansatz functions $w(x, k):=$ $k\left(|x|-\frac{1}{2}\right)$. The parameter $k$ is determined such that $\left|\sigma^{2}\left[w(\cdot, k)-u^{\delta}\right](0)\right|=\lambda_{2}$ (the ansatz function $w$ bends at $x=0$, hence the $\sigma^{2}$ of the dual minimizer has to be extremal, hence equal to $\lambda_{2}$ ). This ansatz function works until, for some $\lambda_{2}=\bar{\lambda}_{2}$, we have $u^{\delta}(0)=w(0, k)$.

- Then for $\lambda_{2} \leq \bar{\lambda}_{2}$, we use a different ansatz function $w$ such that $w(x)=u^{\delta}(x)$ for $x \in(-c, c)$ and some $c>0$ and $w(x)$ is affine linear in $(-1,-c) \cup(c, 1)$ and continuous at $x= \pm c$. The coefficients are determined such that $w \in \mathcal{H}^{2}$ and $\left|\sigma^{2}\left[w-u^{\delta}\right](c)\right|=\lambda_{2}$.

We illustrated both types of solutions in figure 8.1 (right).

8.3. $\mathcal{G}$-minimizers. For $\left(\lambda_{1}, \lambda_{2}\right) \in \Lambda$ as in Definition 5.4, we set up an ansatz function that satisfies the following:

- $w$ is continuous,

- $w(x)=w(-x)$

- $w(x)=u^{\delta}(x)$ for $x \in\left(c_{2}, c_{3}\right)$ and $0 \leq c_{2}<c_{3} \leq 1$,

- either $w$ bends at $x=0$, or $w(x)=u^{\delta}(x)$ for $x \in\left[0, c_{1}\right)$ with $c_{1}<c_{2}$,

- $w$ is piecewise affine linear else.

We illustrate minimizers for different choices of $\left(\lambda_{1}, \lambda_{2}\right)$ in figure 8.1.

\section{Conclusion}

The main motivation of this work has been to show that total generalized variation regularization can be analyzed with the functional analytical framework of the *norm, which has been established in the ingenious book [12] for the Rudin-OsherFatemi functional [16]. Calculating minimizers of some one-dimensional examples 


\section{EXACT SOLUTION OF ONE-DIMENSIONAL TOTAL GENERALIZED VARIATION}

analytically reveals the complicated interplay of the two regularization parameters in TGV minimization.

Acknowledgments. This work has been supported by the Austrian Science Fund (FWF) within the national research networks Photoacoustic Imaging in Biology and Medicine (project S10505) and Geometry \& Simulation (project S11704). C. P. acknowledges support by the Austrian Science Fund (FWF) within the Schrödinger program (project J-297). We thank Carola Schönlieb (Cambridge) for the initiative during the review process to get knowledge of [13].

\section{REFERENCES}

[1] F. Andreu-Vaillo, V. Caselles, and M. Mazón, Parabolic Quasilinear Equations Minimizing Linear Growth Functionals, Progress in Mathematics, Birkhäuser Verlag, Basel, 223, 2004.

[2] G. Aubert and J.F. Aujol, Modeling very oscillating signals. Application to image processing, Appl. Math. Optim., 51, 163-182, 2005.

[3] H.H. Bauschke and P.L. Combettes, Convex Analysis and Monotone Operator Theory in Hilbert Spaces, CMS Books in Mathematics/Ouvrages de Mathématiques de la SMC, Springer, New York, 2011.

[4] M. Benning, C. Brune, M. Burger, and J. Müller, Higher-order TV methods-enhancement via Bregman iteration, J. Sci. Comput., 54, 269-310, 2013.

[5] K. Bredies, K. Kunisch, and T. Pock, Total generalized variation, SIAM J. Imaging Sci., 3(3), 492-526, 2010.

[6] T.F. Chan and S. Esedoḡlu, Aspects of total variation regularized $L^{1}$ function approximation, SIAM J. Appl. Math., 65(5), 1817-1837, 2005.

[7] P.L. Davies and A. Kovac, Local extremes, runs, strings and multiresolution, Ann. Statist., 29(1), 1-65, 2001.

[8] F. Demengel, Problèmes variationnels en plasticité parfaite des plaques, Numer. Funct. Anal. Optim., 6, 73-119, 1983.

[9] F. Demengel, Fonctions à Hessien borné, Ann. Inst. Fourier (Grenoble), 34, 155-190, 1984.

[10] I. Ekeland and R. Temam, Convex Analysis and Variational Problems, North-Holland, Amsterdam, 1976.

[11] E. Mammen and S. van de Geer, Locally adaptive regression splines, Ann. Statist., 25(1), 387-413, 1997.

[12] Y. Meyer, Oscillating Patterns in Image Processing and Nonlinear Evolution Equations, Volume 22 of University Lecture Series, American Mathematical Society, Providence, RI, 2001.

[13] P. Konstantinos and K. Bredies, A study of the one dimensional total generalised variation regularisation problem, arXiv:1309:5900, preprint, 2013.

[14] C. Pöschl, Tikhonov Regularization with General Residual Term, PhD thesis, University of Innsbruck, Austria, Innsbruck, October 2008.

[15] C. Pöschl and O. Scherzer, Characterization of minimizers of convex regularization functionals, in Frames and Operator Theory in Analysis and Signal Processing, Contemp. Math., Amer. Math. Soc., Providence, RI, 451, 219-248, 2008.

[16] L.I. Rudin, S. Osher, and E. Fatemi, Nonlinear total variation based noise removal algorithms, Phys. D, 60(1-4), 259-268, 1992.

[17] O. Scherzer, Denoising with higher order derivatives of bounded variation and an application to parameter estimation, Computing, 60(1), 1-27, 1998.

[18] O. Scherzer, M. Grasmair, H. Grossauer, M. Haltmeier, and F. Lenzen, Variational Methods in Imaging, Appl. Math. Sci., Springer, New York, 167, 2009.

[19] G. Steidl, A note on the dual treatment of higher-order regularization functionals, Computing, 76(1-2), 135-148, 2006. 